\title{
Risk Analysis and Decision Making FY 2013 Milestone Report
}

DW Engel, PNNL

AC Dalton, PNNL

CB Dale, LANL

E Jones, LLNL

J Thompson, LANL

June 2013

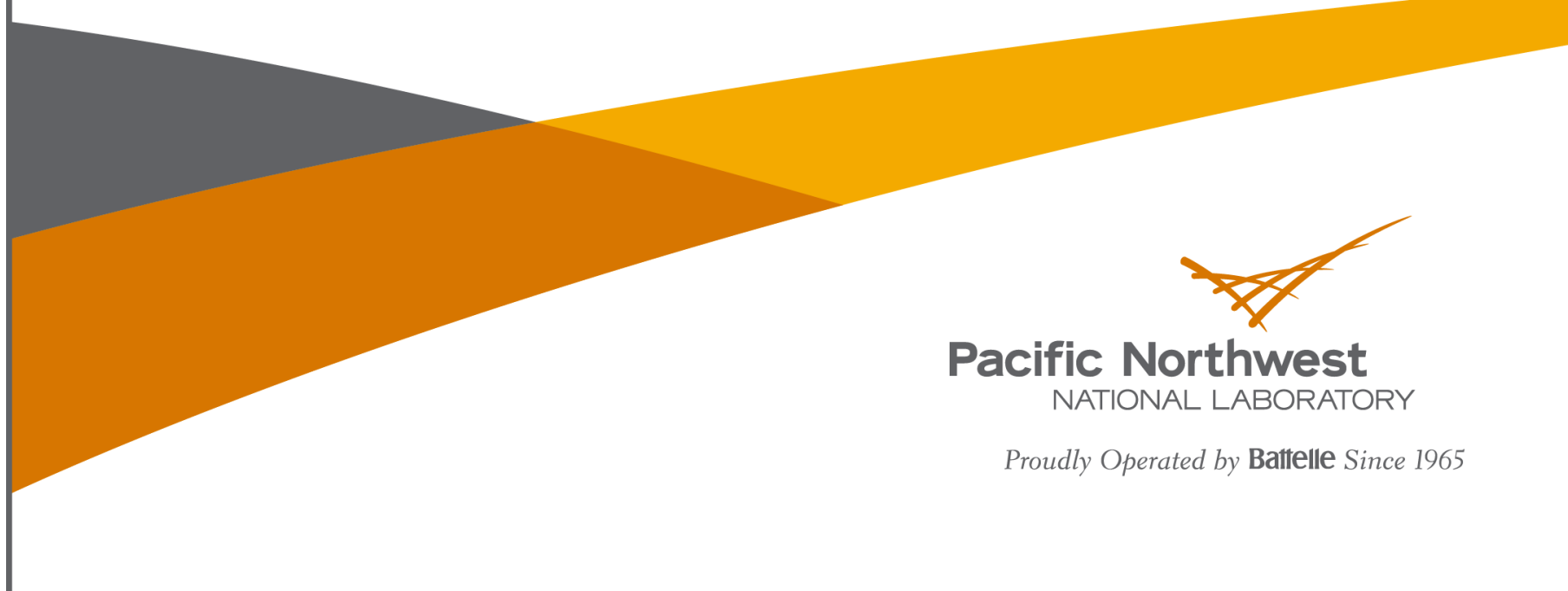




\section{Risk Analysis and Decision Making FY 2013 Milestone Report}

DW Engel, PNNL

AC Dalton, PNNL

CB Dale, LANL

E Jones, LLNL

J Thompson, LANL

June 2013

Work performed under PNNL Project 60174

Prepared by

Pacific Northwest National Laboratory

Richland, Washington 99352

In collaboration with Los Alamos National Laboratory, Lawrence Livermore National Laboratory, and National Energy Technology Laboratory.

Prepared for the

U.S. Department of Energy

National Energy Technology Laboratory 


\title{
DISCLAIMER
}

This report was prepared as an account of work sponsored by an agency of the United States Government. Neither the United States Government nor any agency thereof, nor Battelle Memorial Institute, nor any of their employees, makes any warranty, express or implied, or assumes any legal liability or responsibility for the accuracy, completeness, or usefulness of any information, apparatus, product, or process disclosed, or represents that its use would not infringe privately owned rights. Reference herein to any specific commercial product, process, or service by trade name, trademark, manufacturer, or otherwise does not necessarily constitute or imply its endorsement, recommendation, or favoring by the United States Government or any agency thereof, or Battelle Memorial Institute. The views and opinions of authors expressed herein do not necessarily state or reflect those of the United States Government or any agency thereof.

\author{
PACIFIC NORTHWEST NATIONAL LABORATORY \\ operated by \\ BATTELLE \\ for the \\ UNITED STATES DEPARTMENT OF ENERGY \\ under Contract DE-AC05-76RL01830
}

Printed in the United States of America
Available to DOE and DOE contractors from the
Office of Scientific and Technical Information,
P.O. Box 62, Oak Ridge, TN 37831-0062;
ph: (865) 576-8401
fax: (865) 576-5728
email: reports@adonis.osti.gov

\author{
Available to the public from the National Technical Information Service, \\ U.S. Department of Commerce, 5285 Port Royal Rd., Springfield, VA 22161 \\ ph: (800) 553-6847$$
\text { fax: (703) 605-6900 }
$$ \\ email: orders@ntis.fedworld.gov \\ online ordering: http://www.ntis.gov/ordering.htm
}




\section{Table of Contents}

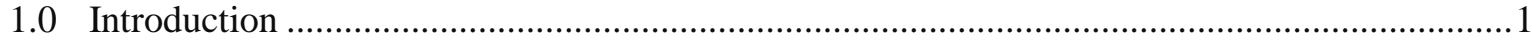

1.1 CCSI Project Overview and Role of Risk Analysis \& Decision Making .......................... 1

1.2 CCSI Risk Analysis \& Decision Making Team: Progress to Date .....................................2

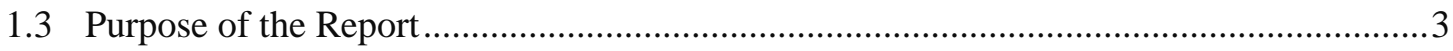

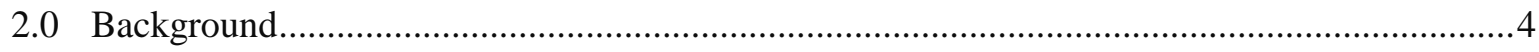

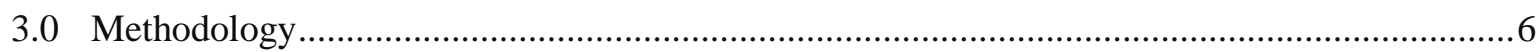

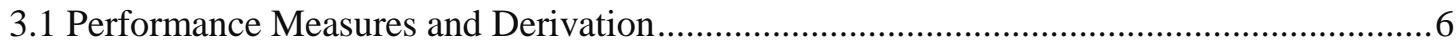

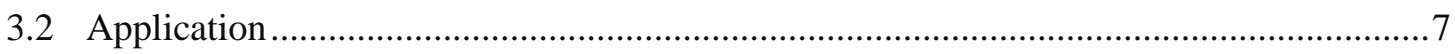

3.2.1 Description of Application Case: 1 MW Hybrid Solid Sorbent System .................. 8

3.2.2 Hybrid Solid Sorbent Carbon Capture System Reliability Block Diagram .............9

4.0 Risk Analysis and Preliminary Results.......................................................................... 11

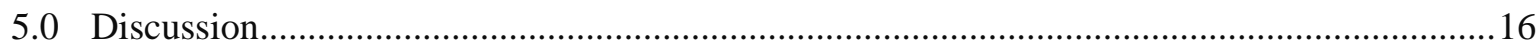

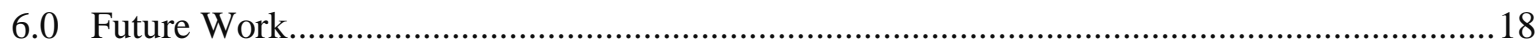

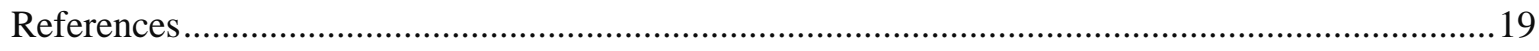

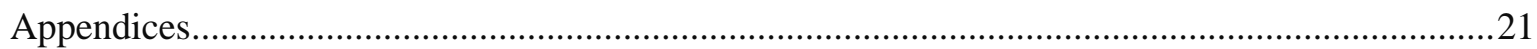

Appendix A: Review of Carbon Capture System Components and Functions .......................21

Appendix B: Definition of Performance Criteria and Risk Measures ......................................24

Appendix C: Carbon Capture System Functional Analysis..................................................2 27

Appendix D: Failure Modes and Effects Identification........................................................ 30

Appendix E: Candidate Risk Assessment Methods................................................................ 33

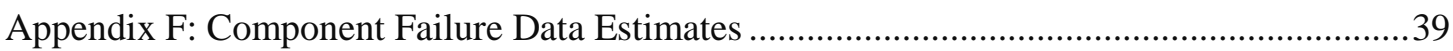

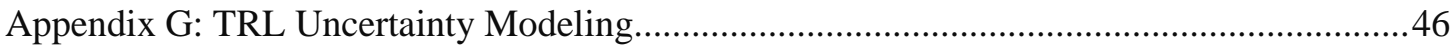

\section{List of Figures}

Figure 1 Process Flow Diagram of the Porposed Solid Sorbent System ..................................... 8

Figure 2 Hybrid Solid Sorbent Carbon Capture System Reliability Block Diagram.................... 10

Figure 3 Reliability Block Diagram for the Adsorber Process ................................................... 11

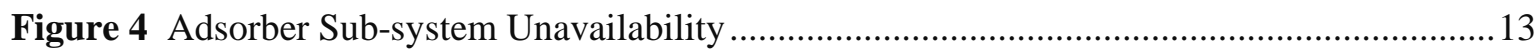

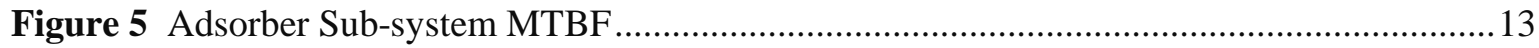

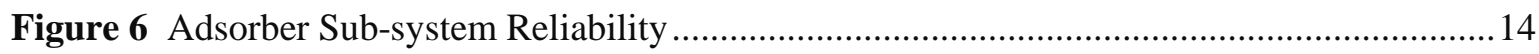

Figure 71 MW Hybrid Solid Sorbent Carbon Capture System Unavailability .............................15

Figure 81 MW Hybrid Solid Sorbent Carbon Capture System MTBF .......................................15

Figure 91 MW Hybrid Solid Sorbent Carbon Capture System Reliability ................................. 15

\section{List of Tables}

Table 1 RMA Analysis Spreadsheet: Adsorber Sub-system ........................................................ 12

Table 2 RMA Analysis Spreadsheet: 1 MW Solid Sorbent Carbon Capture System .................... 14 


\subsection{Introduction}

\subsection{CCSI Project Overview and Role of Risk Analysis \& Decision Making}

Successful development and wide deployment of thermodynamically efficient, cost-effective, and scalable carbon capture technologies provide a potentially paradigm-shifting scientific solution to the carbon dioxide emission problem. However, current carbon capture technologies remain "expensive, cumbersome, and energy-intensive" (DOE 2010). Research suggests that scaling up existing carbon capture technologies for commercial use could increase the cost of electricity by nearly $80 \%$ in new pulverized coal-fired power plants while reducing the plants' net efficiency by about $30 \%$ (Ciferno et al. 2009). Exorbitant costs and lack of efficiency is severely crippling the adoption and deployment of carbon capture technologies by the power industry. Conventional technology development programs in similar technology domains, such as nuclear power generation and the liquefied natural gas industry, often have taken decades before reaching large-scale deployment and maturity, mostly due to intensive testing required at various stages of the development process (Rai et al. 2009). Another time-consuming component of technological development is scalability testing conducted iteratively at increasingly elevated scales before satisfying the requirements of commercial use. The non-linear nature of technology development trajectories suggests that accelerated technology development is possible. For instance, previous research points out that skipping an intermediate scale could result in reduction in development time by about five years (Hirooka 2006).

To tackle the grand scientific challenge of accelerating carbon capture technology development, the Carbon Capture Simulation Initiative (CCSI) established cross-sector collaboration by bringing government, research institutions, and the energy industry together to collectively address barriers to accelerated development, demonstration, and adoption of state-of-the-art carbon capture technologies. By doing so, CCSI seeks to revolutionize the scientific discovery process by investing heavily in advanced multi-scale modeling and simulation $(M \& S)$ of carbon capture technology options. This emphasis has been proven to lead to shortened development time (Hules and Yilmaz 2002). Furthermore, CCSI extends basic M\&S concepts by developing a multi-purpose toolset to enable the development of comprehensive models ranging from the scale of laboratory experiments to full commercial plant scale, facilitate information exchange among designers and decision makers, and provide rigorous quantification of risk and uncertainty to support investment and adoption decisions by the power industry.

Risk analysis and decision making is one of the critical objectives of CCSI, which seeks to use information from science-based models with quantified uncertainty to inform decision makers who are making large capital investments. The goal of this task is to develop tools and capabilities to facilitate the development of risk models tailored for carbon capture technologies, quantify the uncertainty of model predictions, and estimate the technical and financial risks associated with the system. This effort aims to reduce costs by identifying smarter demonstrations, which could accelerate development and deployment of the technology by several years.

Ultimately, the CCSI Risk and Decision Making Team (referred to as the Risk Analysis Team hereafter) will provide a tool suite to enable industry partners to make informed technology adoption and investment decisions. The tool suit will consist of a set of rigorously tested and validated models, uncertainty quantification (UQ), optimization, risk analysis and decision making support capabilities. Commercial and open source software tools employed by the industry will be incorporated to facilitate technology and knowledge transfer. New software will be developed and made available to address technology gaps and strengthen user confidence in technology adoption and incentivize technology diffusion. The resulting CCSI toolset will drive accelerated technology development and adoption in four ways. The toolset will: 1) enable identification of promising concepts, necessary devices and scientific processes through rapid computational screening; 2) lead to the reduction in design and troubleshooting time for new devices and 
processes, 3) make it possible to quantify technical risk as technology maturity transitions from laboratory-scale to commercial-scale; and 4) support reliable estimation of the return on investment and diminished deployment costs by meeting the needs of physical operational testing with high-fidelity simulation of power plants.

\subsection{CCSI Risk Analysis \& Decision Making Team: Progress to Date}

To date, the risk analysis team has developed a prototype demonstration tool that quantifies risk based on the expected profitability of investments to retrofit carbon capture technology on a stylized 650-MWe pulverized-coal electric power generator. The prototype is based on the selection of specific technical and financial factors recommended by industry experts as important determinants of the expected profitability of carbon capture, subject to uncertainty. The uncertainties surrounding the technical performance and the selected financial variables are propagated in a model that calculates the expected profitability of investments in carbon capture and measures risk in terms of variability in expected net returns from these investments.

The current focus of the risk analysis team is in the areas of technology maturation, technical risk analysis, and the coupling of the risk analysis tools. Specifically, the Risk Analysis Team is currently working to complete the following three deliverables:

\section{- TRL-Based Uncertainty Tool}

In this subtask, we are developing an expert elicitation system to assess carbon capture technology readiness levels (TRLs) and use technical maturity information to estimate the level of uncertainty for risk simulations (see Appendix $\mathrm{G}$ for a technical discussion on the uncertainty model). The TRL tools will also include additional models to help determine exit criteria from each TRL, including risk-factor analysis to identify the steps having the greatest impact on risk (schedule, cost, etc.) and links to the stage-gate process typically used in industry for managing technology and product innovation.

\section{- Technical Risk Model (TRM)}

Under this subtask, we are developing a technical risk model that can be used to determine the risk of a proposed technical process and/or design to identify potential failure modes and effects. The results of the technical risk model will feed into an overarching decision-making framework. Products being developed under this subtask consist of four related modules that support:

1. the identification and qualification of risk contributors;

2. the quantification of stochastic distributions for significant risk contributors;

3. risk assessments for defined scenarios, and

4. the integration with process simulations and results from optimization and UQ.

Because the carbon capture technology development process models both mechanical and phenomenological systems, the risk analysis cannot provide estimates of discrete failures (in time) alone; it must also include probabilistic measures of off-normal performance over time. In the current stage of risk analysis, a $1 \mathrm{MW}$ hybrid solid sorbent capture system design is analyzed as the basis for examining risk-based deviations to important decision and financial model parameters. The important phenomena analyzed included the properties and behaviors of the sorbent flows and the mechanical performance of the components containing those flows. The probabilistic measures will include both probabilistic risk assessment (PRA) and uncertainty quantification (UQ) estimations. The risk spectrum can be translated into heuristic distributions for stochastic parameters in the decision framework. The technology risk model can then be coupled with the financial risk model encapsulating economic risk factors and uncertainty to quantify financial returns measures including expected profitability, variability in expected return on investment (ROI), and net present value 
(NPV) estimations. Upon project completion, the risk analysis team will provide both basic risk assessment tools and a portfolio management template for fusing numerical surrogate information into mature financial risk assessment models.

\section{- Decision-Making Framework}

The Risk Analysis Team is developing risk management decision tools, in parallel with numerical simulation capabilities and uncertainty quantification efforts, to directly address technical and financial risks associated with carbon capture technology through the creation of the Risk Analysis \& Decision-Making Framework. The framework consists of a technical risk model (TRM) and a financial risk model (FRM), and requires the translation of technical and financial risk factors, along with uncertainties, into the estimation of variability in expected financial returns. The TRM incorporates multiple risk contributors including technology readiness and technical scale-up risk, as well as uncertainty in models and simulations provided by other CCSI element teams. Many inputs into the financial risk model (FRM) are technical in nature and will also be represented in the TRM. Some of these parameters will come from the process synthesis and design modeling (Process Modeling Team), with uncertainties being simulated by the modeling team and input distributions and uncertainty analysis methods defined by the UQ Team. The marginal distributions for these technical risk factors will then be input into the FRM. The resulting risk analysis will be able to support the (accelerated) development of reliable and cost effective carbon capture systems, by providing evaluation of current vulnerabilities, strategies for improvements, and inputs for on-going tradeoff analyses among decision variables. A wiki-based system will be developed for users to access all of our risk models within a single system and graphic user interface (GUI). This system will automatically pass information (results) between models to synthesize, analyze, report, visualize and display key risk analysis results.

\subsection{Purpose of the Report}

This report documents the technical risk modeling effort based on an initial carbon capture risk analysis to demonstrate the risk modeling and decision support capabilities being developed by the project team. It satisfies the final Year 2 milestone for the Risk Analysis Team/Product: Risk analysis \& decision making of the CCSI program. This milestone is defined as the following:

\section{Milestone: Report documenting the application of the initial risk analysis methodology for the 1 MW solid sorbent based carbon capture system (A1.1)}

In the following sections, we provide an overview of the background and context of the research effort, and then discuss the conceptual approach, methodology, and an initial demonstration of the methodology through a case study using a $1 \mathrm{MW}$ hybrid solid sorbent system. It warrants highlighting that the goal of the Risk Analysis Team is to develop and provide computational and simulation tools, methods, and capabilities that are tailored specifically for the development of carbon capture technologies. It is our hope that these research products will enable future developers of carbon capture technologies to perform rapid, efficient, and robust technical and financial risk analysis.

Therefore, the application example, quantitative analyses, and preliminary results presented in this report are intended primarily as a demonstration to illustrate our current progress in methodological conceptualization and analytical approach. Thus, it is crucial to recognize that:

1. the carbon capture technology system on which we based our analytical approach is still under development, severely limiting the amount of information available for our risk analysis demonstration; 
2. the underlying assumptions of the method used in the demonstration are substantially simplified for the purpose of conceptual parsimony; and

3. many of the parameter values used in our model are leveraged from published sources designed for other relevant industrial domains and/or based on engineering judgment.

Consequently, a literal interpretation of the quantitative results and the presumption that the analysis is empirically grounded and validated are strongly discouraged.

\subsection{Background}

Risk, classically defined by Kaplan and Garrick (1982) as a triplet, is operationalized as the answers to three fundamental questions: 1) possible risk scenarios (what can go wrong?), 2) likelihood of these scenarios, and 3) consequences. Risk analysis and decision-making activities typically focus on two key elements of risk management, namely, technical risk (e.g., will process and plant performance be effective, safe, and reliable?) and enterprise risk (e.g., can project losses and costs be controlled within the constraints of market demand to maintain profitability and investor confidence?). Approaches to risk analysis and assessment abound. Adams (1995) grouped risk evaluation into two broad categories: formal and informal approaches. While formal approaches are based predominantly on scientific, objective identification and assessment of risk factors and related consequences based on rigorous modeling, informal risk evaluation focuses on the socially constructed potential outcomes and consequences of risk for humans, institutions, and social systems (Renn 1992, and Wildavsky and Dake 1990). Within the formal risk evaluation tradition, risk analysis techniques differ in their objectives and technical sophistication (Renn 1992). The actuarial approach, widely used in the insurance industry, measures expected risk as an arithmetic mean of the occurrences of risk events that cause observable and measurable injuries to people and/or their environment. This approach does not seek to identify the causal mechanisms underlying event occurrences. By comparison, the goal of the toxicological or epidemiological approach to risk analysis is to unravel causal explanations of risk through modeling the theoretical assumptions, drivers, and conditions that might have precipitated the emergence of the events. Rather than treating risk as the embodiment of individual events, the probabilistic approach to risk analysis espouses a systemic perspective. This approach tends to utilize techniques such as fault trees or decision trees to decompose the system in question into individual components, and then generate a stochastic risk estimate for each component based on quantifiable observations. This probabilistic approach not only offers risk information at a finer granularity but also produces a risk-informed view of the system as a whole.

Existing research suggests a broad spectrum of risk domains, including technology (Klein and Cork 1998), product development (Wu et al. 2010), regulatory environment (Pennington et al. 2006), market (Alexander 2009), cost (Balci and Sargent 1981), strategy (Miller and Lessard 2008), schedule (Browning and Eppinger 2002), and planning and communication (Kwak and Stoddard 2004; Lundgren and McMakin 2011). Wu et al. (2010) usefully assign these various risks into two groups.

1. External risks, such as risk in the regulatory environment and the market, include those domains beyond the sphere of influence of the individual or organization.

2. Internal risks, such as technical risk and cost, capture the risk domains that can be mitigated through effective risk management practices.

In the context of technology development project management, managing risk refers to proactive actions undertaken to identify and obviate the occurrences of undesired project outcomes, and to mitigate negative consequences in the wake of an undesired event (Smith and Merritt 2002). Time, cost, and performance, thus, are the three central concerns of risk management (Chapman 1990). According to the technology risk quantification model developed by Hartmann and Myers (2001), four levels of technical 
risk exist for technology development projects, depending on the degrees of technological novelty and market potential. These risks range from

1. evolutionary risk (lowest risk with existing markets and technology),

2. leverage base risk (medium risk with new markets and existing technology),

3. discontinuity risk (medium risk with existing markets and new technology), to

4. radical risk (highest risk with both new markets and new technology).

As a project aimed at developing large-scale and highly complex new technologies geared toward an emerging market, the Carbon Capture Simulation Initiative falls into the "radical" risk category, and thus is especially sensitive to risks associated with development lead time, development and deployment cost, and technology performance.

Since development time and cost are substantially influenced by technical risk and system performance, we focus on these two domains in our risk analysis and assessment activities. Specifically, technical risk assessment estimates "the likelihood that the system embodied in a design will, when constructed, meet the performance requirements it is intended to meet, and if a shortfall in performance is expected, how serious the shortfall is likely to be" (Klein and Cork 1998, p. 345). Following Modarres (2006), we define system performance as "the ability of the system to realize its intended functions at all times" (p. 18), which is conditioned on the system's capacity/strength, stress tolerance, and damage endurance. System performance failure can thus be estimated stochastically by quantifying the cumulative and interactive effects among strength, stress, and damage measures, and by analyzing how failure might result from any one or combinations of the three performance metrics. In addition to capability, efficiency and availability are also important performance indicators. While efficiency is often quantified by comparing system input and output, availability refers to probability that the system might be down "due to incipient failure," preventing the system from performing its expected functions (Modarres 2006, p.19). Unobserved component failure, repair, and maintenance are frequent contributors to unavailability (Zio 2007). System availability can be further represented by reliability and maintenance. Reliability is often operationalized as the probability of the technology staying in a non-failure state over "an N-year planning period" (Xu et al. 1998, p. 21). Coupled with reliability, performance risk can also be examined by resiliency (measured as the probability of the technology being restored to normal operation following a failure) and vulnerability (measured as the significance of the failure (Xu et al. lbid.). If performance can be directly observed, another approach to modeling performance risk can be accomplished through modeling performance degradation over time. By estimating the distribution of system performance over time against a predetermined performance acceptance floor, be it a discrete value or a distribution, performance failure can then be modeled as the probability that the system or a unit in question fails to meet the specified minimum performance requirement criterion at a point in time (See Appendix B for a detailed discussion on definition of performance criteria and risk measures). Failure model and effects analysis (FMEA), failure modes, effects, and criticality analysis (FMECA), relative risk profiling, and scenario-based risk analysis with Monte Carlo simulations are used extensively in technical risk analysis. A detailed description of these methods and applications is provided in Appendix E. Significant expertise in applying these methods to various risk domains is represented on the CCSI Risk Analysis Team (see, for example, Pawar et al. 2013; Jones et al. 1995; Engel 2012; Scott et al. 2005; Kincaid et al. 2002; Dale, et al. 2006).

Guided by the indicated literature, the current risk analysis effort focuses on quantifying risks associated with a hybrid solid sorbent carbon capture system availability, and investigates the extent to which performance is shaped by availability of system functional components. In this report, the estimate of operational availability of the carbon capture system is based on an estimate of the mean time between failure (MTBF) and mean down time (MDT) of the system. In practice, these variables are obtained by summation of component failure and repair rates with consideration for the multiplicity of each equipment type and any redundancy that exists. A reliability block diagram (RBD) was developed to structure the summation of component failure, and assumptions about repair rate and redundancy are based on surrogate engineering systems (or have been ignored for the sake of parsimony). Leveraging a number of the risk approaches discussed in this section as well as in the appendices, the technical risk 
analysis capability that the Risk Analysis Team is developing focuses on identifying and quantifying those factors having the greatest impact on the estimated reliability of the system performance with the hope to better facilitate the development of a carbon capture system. It warrants emphasizing that the goal of CCSI is to advance and accelerate the creation and deployment of a new carbon capture technology that will satisfy or even exceed the performance criteria currently based on energy and profit loss due to the addition of the CC system. We elaborate on CCSI technical risk analysis approaches below.

\subsection{Methodology}

For the initial risk analysis, we conducted a reliability, maintainability, and availability (RMA) analysis using a 1-MW hybrid solid sorbent system as the application case. This preliminary analysis demonstrates our current methodological conceptualization and proof-of-concept implementation of carbon capture technical risk analysis. In Section 3.1, we define the system performance measures employed in our analysis, and provide the mathematical derivation for RMA calculations. In Section 3.2, we describe the application of the RMA analysis in the context of our foundational risk analysis conceptual framework, which is based on the tight integration between the solid sorbent system process design and a corresponding, simplified reliability block diagram (RBD) representation of performance risk.

\subsection{Performance Measures and Derivation}

We use reliability, maintainability, and availability as measures for system performance measures. These terms are defined as follows.

Reliability, also called probability of survival is the probability that an item will perform its intended function over a desired time period, without failure, under specified conditions, with a desired confidence. Describing a complex system in terms of its reliability alone is not, however, sufficient to characterize the system's performance. Reliability needs to be assessed in concert with the overall maintainability and availability of a system.

Maintainability includes actions implemented to restore a failed item to full function as well as preventative maintenance and sparing strategies, which serve to prevent item failure from impacting system performance.

Availability is defined as the probability that a system will be operating properly during the times that it is scheduled to operate. Its complement, unavailability, is often referred to as the probability of failure to operate on demand. Unlike reliability, availability accounts for the time to repair a failed item and return to operation.

For the purposes of this analysis, we will consider that equipment failures are random and independent of component age, exhibiting a constant failure rate for each component. For a more detailed discussion of the nature of failure rate as a function of age, please see Appendix B. Considering the constant failure rate region of equipment life, ignoring infant mortality and wear out (aging) failures, the corresponding reliability of an individual component is exponential, with a constant failure rate $(\lambda)$ of a component. The corresponding reliability, $\mathrm{R}$, of that component may be expressed by,

$$
R=e^{-\lambda \mathrm{t}}
$$

For repairable systems, the inverse of failure rate, $(1 / \lambda)$, is referred to as the mean time between failure (MTBF). MTBF is a common data format used for reliability analyses. For maintenance estimates, a constant restoration time is used to estimate the time required to return the component (system) to operational status after a failure. Where all downtime is included, the restoration time is referred to as the mean down time (MDT). Using these symbolic representations, we may express Operational Availability, $A_{o}$ as: 


$$
A_{o}=M T B F /(M T B F+M D T)
$$

The risk measure that we will focus on for this analysis is unavailability, $\left(1-A_{o}\right)$. Unavailability, also called probability of failure on demand (PFD), describes the proportion of time for which an item is failed and cannot be used. In reliability work, it is usually referred to as unavailability because the word describes the situation of the plant, i.e., not producing. In safety-related work, where a safety function is inhibited, the PFD term is more common. Unavailability is the ratio of downtime to total time,

$$
\mathrm{UnA}=\mathrm{MDT} /(\mathrm{MTBF}+\mathrm{MDT})
$$

Replacing MTBF with $1 / \lambda$, for constant failure rates, then multiplying both the numerator and denominator by $\lambda$, the unavailability of the system can be expressed as

$$
\mathrm{UnA}=\lambda \mathrm{MDT} /(1+\lambda \mathrm{MDT})
$$

To put this expression in context, recall that risk is defined as the product of likelihood and consequence. In this analysis, likelihood is the component failure rate and consequence is the MDT. Thus, Unavailability, being a function of the product of the component failure rate and the MDT, is an effective risk metric.

Recalling that for constant failure rates, the reliability for each component is given as:

$$
R_{i}=e^{-\lambda_{i} t}
$$

The reliability of the system, which also has a constant failure rate, may be expressed in the same form,

$$
R_{S}=e^{-\lambda_{S} t}
$$

Provided that the two assumptions of constant failure rate and series modeling apply, then it is valid to speak of a system failure rate computed from the sum of the individual unit or component failure rates (see Section 5 for a discussion on the rationale for adopting these assumptions).This assumption holds for each function block as well as for the aggregate functions making up a subsystem or the whole system, e.g., the failure rate for each function block is the sum of the failure rates of its constituent components. Where $\lambda_{\mathrm{s}}$ is the sum of the individual failure rates, the system reliability is described as:

$$
R_{S}=e^{-\left(\sum_{i} \lambda_{i}\right) t}
$$

The practice of adding up the component failure rates assumes that any single failure causes a system failure and entails some down time. This assumption is therefore a worst-case prediction. Under these assumptions, the UnA for the system is predicted as:

$$
U n A_{s}=\frac{\sum_{i} \lambda_{i} M D T_{i}}{\sum_{i}\left(1+\lambda_{i} M D T_{i}\right)}
$$

The prescription is then to estimate the failure rate and MDT for each component under each function whose failure causes a system failure, from which the failure rate, MTBF, MDT and unavailability of any combination of components can be calculated.

\subsection{Application}

Ultimately, the risk analysis team will tackle carbon capture technology risk, a multi-dimensional risk phenomenon, by providing comprehensive stochastic risk modeling and simulation tools and capabilities, coupled with uncertainty quantification. However, in the near term, we focus on one of the most basic and yet crucial risk dimensions - functional reliability for a $1 \mathrm{MW}$ hybrid solid sorbent system - to shed light on how reliability affects the overall performance failure in carbon capture technologies. This focus, while effectively addressing analytical necessity and coping with the current epistemic constraints, boasts a number of methodological advantages. First, for emerging and complex technologies like carbon capture systems, a substantial body of technical details about the systems remains unspecified or underspecified, making a comprehensive risk analysis at the current stage of research and development 
impractical. Thus, we focus on the arguably better understood reliability aspect of the carbon capture system and have based the initial analysis on those functional components that are relatively more defined. Second, related to the newness of the technology is the persistent lack of system data. As a means of circumventing this challenge, functional failure analysis allows the researchers to leverage the basic assumptions, parameter values, and risk quantification techniques from other related technology domains to estimate how they might influence the likelihood, magnitude, and consequences of functional risk in carbon capture systems. Third, in view of the next phase of the risk analysis research with the objective to incorporate additional non-technical risk factors into the risk models, the functional failure risk analysis discussed below will provide a foundational, overarching risk analysis building block to facilitate the addition of risk factors from the financial risk model and UQ modeling and simulation. Thus, focusing on modeling functional failure and reliability as it relates to meeting system performance goals serves as a calculated, strategic launching point for more comprehensive risk analysis in the near future.

\subsubsection{Description of Application Case: 1 MW Hybrid Solid Sorbent System}

The conceptual design for the 1-MW carbon capture system comprises two main systems: a two-stage fluidized adsorber bed, and a moving bed regenerator (Lee et al. 2012a; Lee et al. 2012b) (see Figure 1). A more detailed description of the system is provided in Appendix A.

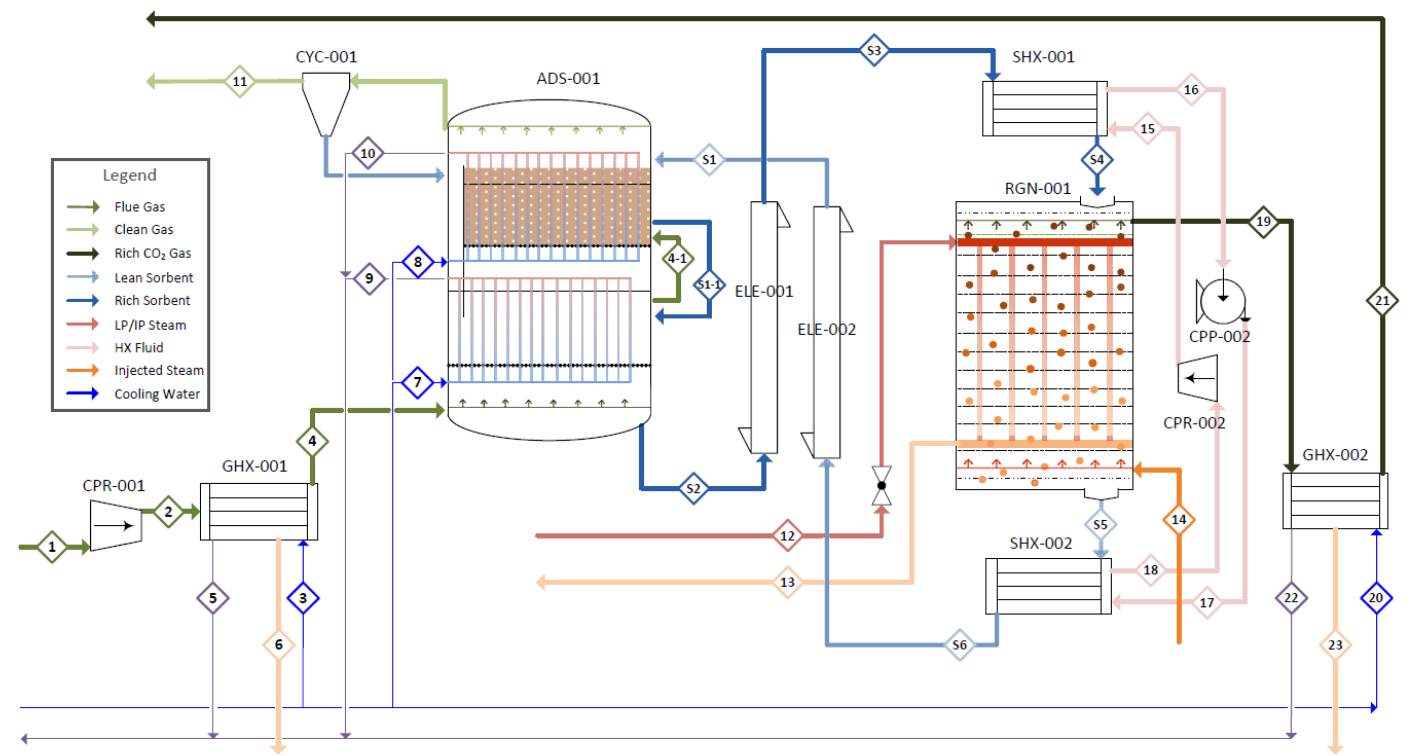

Figure 1 Process Flow Diagram of the pPrposed Solid Sorbent System

The process flow, as defined for this report, begins with introduction of flue gas consisting of carbon dioxide, water, and nitrogen, and ends with compression of $\mathrm{CO}_{2}$ for pipeline transport. The system can be further broken out into five major functions:

- Preparation of flue gas

- Carbon dioxide adsorption

- Loaded sorbent transport to regenerator and regenerated sorbent transport to adsorber

- Sorbent regeneration

- Carbon dioxide recovery 


\subsubsection{Hybrid, Solid Sorbent Carbon Capture System Reliability Block Diagram}

For the analysis of the carbon capture system, we developed a reliability block diagram (RBD) as the modeling apparatus. Generally, a reliability block diagram (RBD) describes the system as a number of functional blocks that are interconnected according to the effect that each block has on the overall system reliability. To generate this diagram, we implemented a conceptual parsimony mapping exercise to superimpose the process flow of a model carbon capture system (Figure 1) on a linear RBD to specify major functional/risk components (as blocks) at a coarse-grain level and sub-components within each block to depict the serial and multi-tiered relationships among various system functions. This mapping allows for a tighter analytical integration between system process and system reliability assessment.

As Figure 2 illustrates, the process flow can be mapped into a series of functional blocks. Each block represents a number of components in order to simplify the system so that each block is easily identified. Note that these blocks should be mutually independent in that failure of one block will have no influence over the probability of failure in another. For a series diagrammatic configuration like Figure 2, blocks should not contain any significant redundancy so that the addition of failure rates of components within a block is valid. Within each block, specific functional components are enumerated. As Figure 3 demonstrates, within each block, the specific functional components can be further analyzed and organized into one or more finer-grained process flows that fulfill the high-level functional expectations. For this initial risk analysis, only the adsorber process (functional block) has been further divided into its finer-grained process flow.

For the Hybrid Solid Sorbent RBD, we define failure as non-conformance to some specified performance criterion (see Appendix $\mathrm{C}$ for the list of performance criteria and associated assumptions). And thus, failed states are assumed to be binary, i.e. failed or not failed, for equipment and phenomena (e.g., carbon loading). For the initial analysis, failure rates are assumed to be constant and independent. It follows that for the hybrid solid sorbent superstructure design, a series of RBD is applicable such that failure of any block prevents the operation of the system. Hence, the estimation of failure rate and mean down time (MDT) of each component under each function block allows a calculation of mean time between failures (MTBF), MDT, and unavailability for combinations of components, each block, combinations of blocks, or for the whole system. 


\begin{tabular}{|c|c|c|c|c|}
\hline $\begin{array}{c}\text { Prepare Flue } \\
\text { Gas }\end{array}$ & $\begin{array}{l}\text { Adsorb } \mathrm{CO}_{2} \\
\text { from Flue Gas } \\
\text { by Sorbent } \\
\text { (Adsorber) }\end{array}$ & $\begin{array}{l}\text { Sorbent } \\
\text { Transport - } \\
\text { Pneumatic } \\
\text { System }\end{array}$ & $\begin{array}{l}\text { Desorption of } \\
\mathrm{CO}_{2} \text { from } \\
\text { Sorbent } \\
\text { (Regenerator) }\end{array}$ & $\begin{array}{c}\text { Prepare } \mathrm{CO}_{2} \\
\text { for } \\
\text { Compression } \\
\text { Train }\end{array}$ \\
\hline $\begin{array}{l}\text { - Flue gas composition } \\
\text { - Compressor } \\
\text { - Gas heat exchanger } \\
\text { - Gas piping (3) } \\
\text { - Gas valves (2) } \\
\text { - Gas valve } \\
\text { controller(2) } \\
\text { - Water pump } \\
\text { - Water piping (3) } \\
\text { - Water valves (3) } \\
\text { - Water controller (2) }\end{array}$ & $\begin{array}{l}\text { - Cooling water piping (3) } \\
\text { - Water valves (3) } \\
\text { - Water valve controller (3) } \\
\text { - Water pump } \\
\text { - Flue gas pipe/inlet } \\
\text { - Gas valve } \\
\text { - Gas valve controller } \\
\text { - Clean gas pipe/outlet } \\
\text { - Clean gas valve } \\
\text { - Clean gas controller } \\
\text { - Cyclone unit and fines } \\
\text { return } \\
\text { - Sorbent in and out: } \\
\text {-piping (2) } \\
\text {-valves (2) } \\
\text {-level controllers (2) } \\
\text { - Upper HX } \\
\text { - Lower HX } \\
\text { - Passive mechanical } \\
\text { structures (failure modes: } \\
\text { fatigue, erosion, } \\
\text { corrosion) } \\
\text { - Carbon loading (failure } \\
\text { modes: flow rates, } \\
\text { selectivity) }\end{array}$ & $\begin{array}{l}\text { - Sorbent piping (4) } \\
\text { - Sorbent valve (4) } \\
\text { - Sorbent valve } \\
\text { controller(4) } \\
\text { - Transport gas piping (4) } \\
\text { - Gas valve (2) } \\
\text { - Gas valve controller (2) } \\
\text { - Compressor (2) } \\
\text { - Air cooler (2) } \\
\text { - Pneumatic pipe (solids } \\
\text { and gas) (2) } \\
\text { - Sorbent collection } \\
\text { hoppers (2) }\end{array}$ & $\begin{array}{l}\text { - Sorbent in and out: } \\
\text {-piping (4) } \\
\text {-valves (4) } \\
\text {-level controllers (4) } \\
\text { - Solid heat exchangers (2) } \\
\text { - Compressor } \\
\text { - Centrifugal pump } \\
\text { - Temperature controller } \\
\text { - Plant hot steam: } \\
\text {-piping (2) } \\
\text {-valves(2) } \\
\text {-level controllers (2) } \\
\text { - Mild steam: } \\
\text {-piping (2) } \\
\text {-valve } \\
\text {-valve controller } \\
\text {-blower } \\
\text { - Solid HX fluid (steam/ } \\
\text { water) } \\
\text {-piping(4) } \\
\text {-valves(4) } \\
\text {-level controllers (4) } \\
\text { - Passive mechanical } \\
\text { structures (failure modes) } \\
\text { - Desorption of sorbent } \\
\text { (failure modes) }\end{array}$ & $\begin{array}{l}\text { - Product } \mathrm{CO}_{2} \text { gas } \\
\text { collector } \\
\text { - Gas piping (2) } \\
\text { - Gas valve (2) } \\
\text { - Gas valve controller (2) } \\
\text { - Gas heat exchanger } \\
\text { - Cooling water pump } \\
\text { - Water piping (2) } \\
\text { - Water valve (2) } \\
\text { - Water level controller (2) }\end{array}$ \\
\hline
\end{tabular}

Figure 2 Hybrid Solid Sorbent Carbon Capture System Reliability Block Diagram. Numbers in parentheses refer to the number of physical components (e.g., 3 adsorber water valves). 

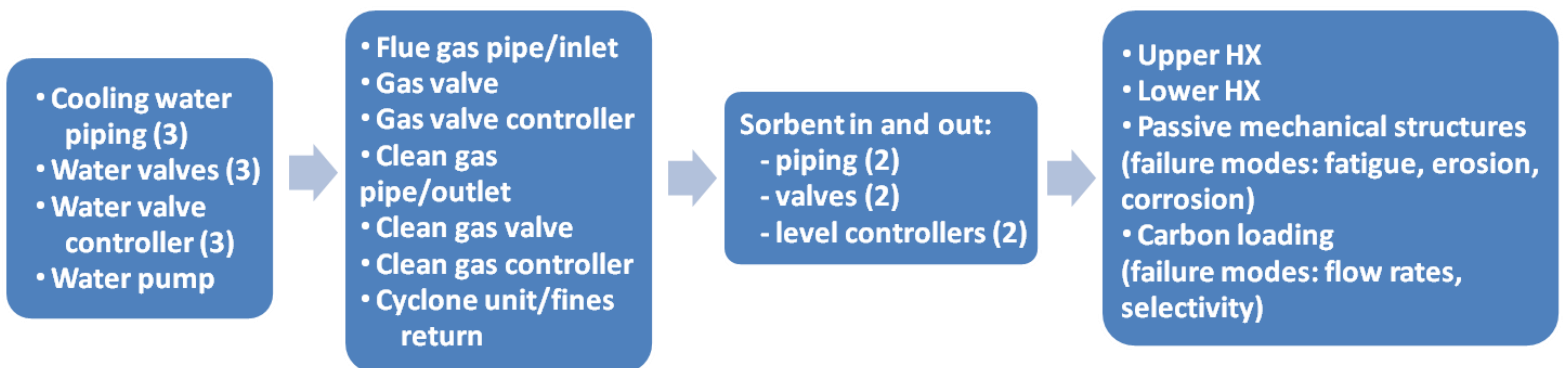

Figure 3 Reliability Block Diagram for the Adsorber Process

\subsection{Risk Analysis and Preliminary Results}

The RMA analysis spreadsheet for the adsorber sub-system is presented Table 1 . The individual component failure rates and associated down times have been assessed as described in detail in Appendix F. The data used is a mixture of generic process industry standards and of engineering judgment where databases are lacking. This usage is appropriate given the under-specified nature of the putative system components. The data sources provide ranges of data, and point estimates are selected from these ranges that are biased towards the mid-range values. It is evident that the numbers selected will dominate the analysis results, and in future studies, the fidelity of data will be enhanced by modeling the potential failure rate and MDT values as probabilistic distributions whereupon confidence estimates can be inferred in stochastic calculations. Therefore, we acknowledge the ambiguity around the utilized data points in this initial deterministic approach, but we believe the data are relevant exemplars and provide analysis results on the discrete relative values of reliability measures.

The spreadsheet captures component-level inputs for failure rate and MDT, with numbers in parentheses indicating the number of identical components reflected by the data, or in the case of piping, the pipe length assumed. The inputs are used to calculate the MTBF, unavailability, and reliability for each component. The components have been organized into rough functional blocks (Figure 3) such that subtotals can be obtained for each functional block if desired.

Note that Table 1 contains information regarding the failure-related adsorption of the sorbent or of the "reliability" of carbon loading. This information was included as a test case to help evaluate the relative impacts of phenomenological chemical process failure issues with mechanical-related failures. The high adsorption failure rate, which dominates the reliability measures, is indicative of the large degree of uncertainty, and low TRL (see Appendix G), in the performance of the sorbent under operating conditions. A lesson-learned is that a reliability approach might not be the most suitable option for dealing with the sorbent performance behavior, but is better treated in a probabilistic approach to meeting performance criteria. The adsorption performance failure has not been included in the overall system reliability rollup. 
Table 1 RMA Analysis Spreadsheet: Adsorber Sub-system

\begin{tabular}{|c|c|c|c|c|c|c|c|}
\hline \multirow{3}{*}{ Adsorber Sub-System } & & & & & \multirow[b]{2}{*}{8,760} & \multirow[b]{3}{*}{$\begin{array}{c}\text { UnA } \\
(\mathbf{h r} / \mathbf{y r})\end{array}$} & \multirow[b]{3}{*}{$\begin{array}{c}\begin{array}{c}\text { Reliability } \\
\text { (annualized) }\end{array} \\
\end{array}$} \\
\hline & \multirow[b]{2}{*}{$\begin{array}{c}\text { Failure rate } \\
\text { (per MHr) }\end{array}$} & \multicolumn{3}{|c|}{ Hours of operation in a year } & & & \\
\hline & & $\begin{array}{c}\text { Failure rate } \\
\text { (per } \mathbf{H r} \text { ) }\end{array}$ & \begin{tabular}{|c|} 
MTBF \\
$(\mathbf{H r})$
\end{tabular} & \begin{tabular}{|c|}
$\begin{array}{c}\text { MDT } \\
(\mathbf{H r})\end{array}$ \\
\end{tabular} & $\begin{array}{c}\text { Probability of } \\
\text { UnA }\end{array}$ & & \\
\hline Cooling Water & & & & & & & \\
\hline Piping - CW (50 m) & 0.03 & $3.1 \mathrm{E}-08$ & $3.2 \mathrm{E}+07$ & 32 & $9.9 \mathrm{E}-07$ & 0 & 1.00 \\
\hline Valves - CW (3) & 4 & 4.2E-06 & $2.4 \mathrm{E}+05$ & 115 & $4.8 \mathrm{E}-04$ & 4 & 0.96 \\
\hline Controllers - CW (3) & 210 & $2.1 \mathrm{E}-04$ & $4.8 \mathrm{E}+03$ & 16 & $3.3 \mathrm{E}-03$ & 29 & 0.16 \\
\hline Pump - CW (1) & 7 & 7.0E-06 & $1.4 \mathrm{E}+05$ & 160 & $1.1 \mathrm{E}-03$ & 10 & 0.94 \\
\hline Subtotal & 221 & $2.2 \mathrm{E}-04$ & $4.5 \mathrm{E}+03$ & 22 & $4.9 \mathrm{E}-03$ & 43 & 0.14 \\
\hline Gas & & & & & & & \\
\hline Piping - G (20 m) & 0.03 & $3.0 \mathrm{E}-08$ & $3.3 \mathrm{E}+07$ & 32 & 9.7E-07 & 0 & 1.00 \\
\hline Valves - G (1) & 3 & $2.8 \mathrm{E}-06$ & $3.6 \mathrm{E}+05$ & 115 & $3.2 \mathrm{E}-04$ & 3 & 0.98 \\
\hline Controllers - G (1) & 140 & $1.4 \mathrm{E}-04$ & $7.1 \mathrm{E}+03$ & 16 & $2.2 \mathrm{E}-03$ & 20 & 0.29 \\
\hline Cyclone unit - G (1) & 9 & $9.0 \mathrm{E}-06$ & $1.1 \mathrm{E}+05$ & 60 & $5.4 \mathrm{E}-04$ & 5 & 0.92 \\
\hline Subtotal & 152 & $1.5 \mathrm{E}-04$ & $6.6 \mathrm{E}+03$ & 20 & $3.1 \mathrm{E}-03$ & 27 & 0.26 \\
\hline Sorbent Transport & & & & & & & \\
\hline Piping - ST (10 m) & 0.05 & $4.5 \mathrm{E}-08$ & $2.2 \mathrm{E}+07$ & 40 & $1.8 \mathrm{E}-06$ & 0 & 1.00 \\
\hline Valves - ST (2) & 4 & $4.2 \mathrm{E}-06$ & $2.4 \mathrm{E}+05$ & 130 & $5.5 \mathrm{E}-04$ & 5 & 0.96 \\
\hline Controllers - ST (2) & 140 & $1.4 \mathrm{E}-04$ & $7.1 \mathrm{E}+03$ & 20 & $2.8 \mathrm{E}-03$ & 24 & 0.29 \\
\hline Subtotal & 144 & $1.4 \mathrm{E}-04$ & $6.9 \mathrm{E}+03$ & 23 & 3.3E-03 & 29 & 0.28 \\
\hline Adsorber Reactor & & & & & & & \\
\hline Upper and lower HX - AR (1 ea) & 64 & $6.4 \mathrm{E}-05$ & $1.6 \mathrm{E}+04$ & 1,000 & $6.0 \mathrm{E}-02$ & 527 & 0.57 \\
\hline Vessel structures - AR (1) & 0.01 & $1.0 \mathrm{E}-08$ & $1.0 \mathrm{E}+08$ & 1,000 & $1.0 \mathrm{E}-05$ & 0 & 1.00 \\
\hline Carbon loading - AR (1) & 3,300 & 3.3E-03 & $3.0 \mathrm{E}+02$ & 50 & $1.4 \mathrm{E}-01$ & 1241 & $2.8 \mathrm{E}-13$ \\
\hline Subtotal & 3,364 & $3.4 \mathrm{E}-03$ & $3.0 \mathrm{E}+02$ & 68 & $1.9 \mathrm{E}-01$ & 1632 & $1.6 \mathrm{E}-13$ \\
\hline Subtotal (ignore carbon loading & 64 & $6.4 \mathrm{E}-05$ & $1.6 \mathrm{E}+04$ & 1,000 & $6.0 \mathrm{E}-02$ & 527 & 0.57 \\
\hline Total & 3,881 & $3.9 \mathrm{E}-03$ & $2.6 \mathrm{E}+02$ & 62 & $1.9 \mathrm{E}-01$ & 1698 & $1.7 \mathrm{E}-15$ \\
\hline Total (ignore carbon loading) & 581 & $5.8 \mathrm{E}-04$ & $1.7 \mathrm{E}+03$ & 130 & $7.0 \mathrm{E}-02$ & 614 & $6.1 \mathrm{E}-03$ \\
\hline
\end{tabular}

The components have been rank ordered (in relative profiles) to demonstrate the critical contributors to risk for the adsorber sub-system as shown in Figure 4. It should be carefully noted that the tornado diagram (i.e. vertically listed bar chart) below has been presented in log scale because the unavailability probability has been normalized to hours per year. The estimates for MTBF and Reliability are similarly presented in Figure 5 and Figure 6. As with UnA, MTBF is shown on a log scale, however, Reliability, which ranges from 0-1, has not. One may see that MTBF and UnA do not agree on the ranking of critical 
risk components. This result is due to the definition of UnA, which accounts for the down time to repair. Some systems with a relatively high failure rate but lower MDT will not be as critical as similar failure rates with longer repair times.

\begin{tabular}{|l|r|}
\hline Component & UnA (hr/yr) \\
\hline Carbon loading - AR (1) & 1241 \\
\hline Upper and lower HX - AR (1 ea) & 527 \\
\hline Controllers - CW (3) & 29 \\
\hline Controllers - ST (2) & 24 \\
\hline Controllers - G (1) & 20 \\
\hline Pump - CW (1) & 10 \\
\hline Valves - ST (2) & 5 \\
\hline Cyclone unit - G (1) & 5 \\
\hline Valves - CW (3) & 4 \\
\hline Valves - G (1) & 3 \\
\hline Vessel structures - AR (1) & 0.09 \\
\hline Piping - ST (10 m) & 0.02 \\
\hline Piping - CW (50 m) & 0.009 \\
\hline Piping - G (20 m) & 0.009 \\
\hline
\end{tabular}

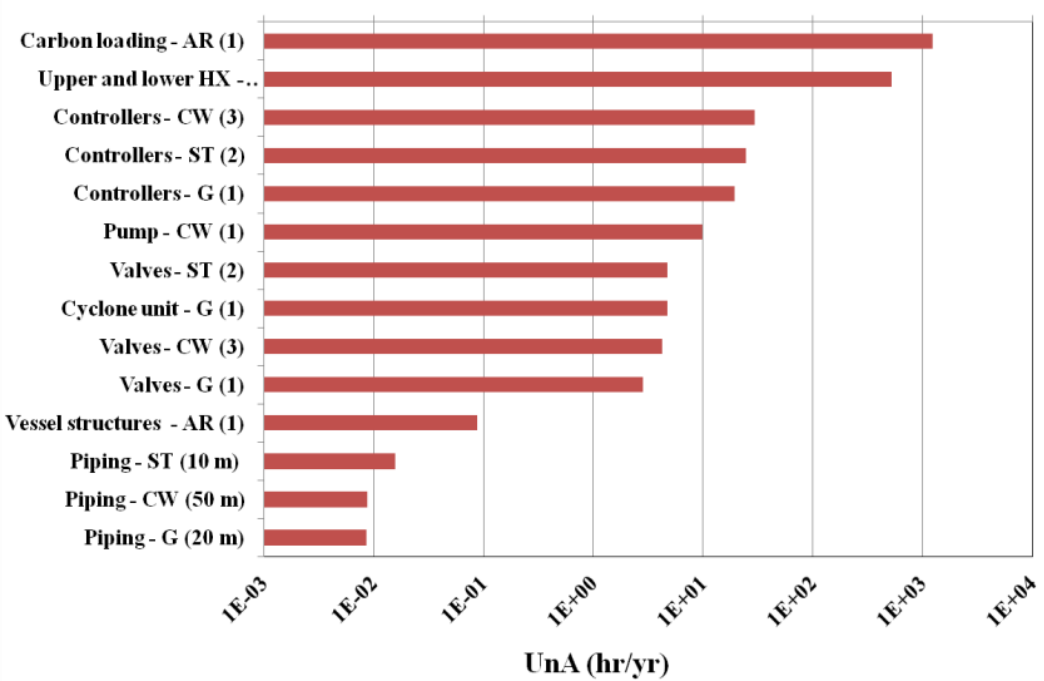

Figure 4 Adsorber Sub-system Unavailability

\begin{tabular}{|l|r|}
\hline Component & MTBF (hr) \\
\hline Carbon loading - AR (1) & $3.0 \mathrm{E}+02$ \\
\hline Controllers - CW (3) & $4.8 \mathrm{E}+03$ \\
\hline Controllers - G (1) & $7.1 \mathrm{E}+03$ \\
\hline Controllers - ST (2) & $7.1 \mathrm{E}+03$ \\
\hline Upper and lower HX - AR (1 ea) & $1.6 \mathrm{E}+04$ \\
\hline Cyclone unit - G (1) & $1.1 \mathrm{E}+05$ \\
\hline Pump - CW (1) & $1.4 \mathrm{E}+05$ \\
\hline Valves - CW (3) & $2.4 \mathrm{E}+05$ \\
\hline Valves - ST (2) & $2.4 \mathrm{E}+05$ \\
\hline Valves - G (1) & $3.6 \mathrm{E}+05$ \\
\hline Piping - ST (10 m) & $2.2 \mathrm{E}+07$ \\
\hline Piping - CW (50 m) & $3.2 \mathrm{E}+07$ \\
\hline Piping - G (20 m) & $3.3 \mathrm{E}+07$ \\
\hline Vessel structures - AR (1) & $1.0 \mathrm{E}+08$ \\
\hline
\end{tabular}

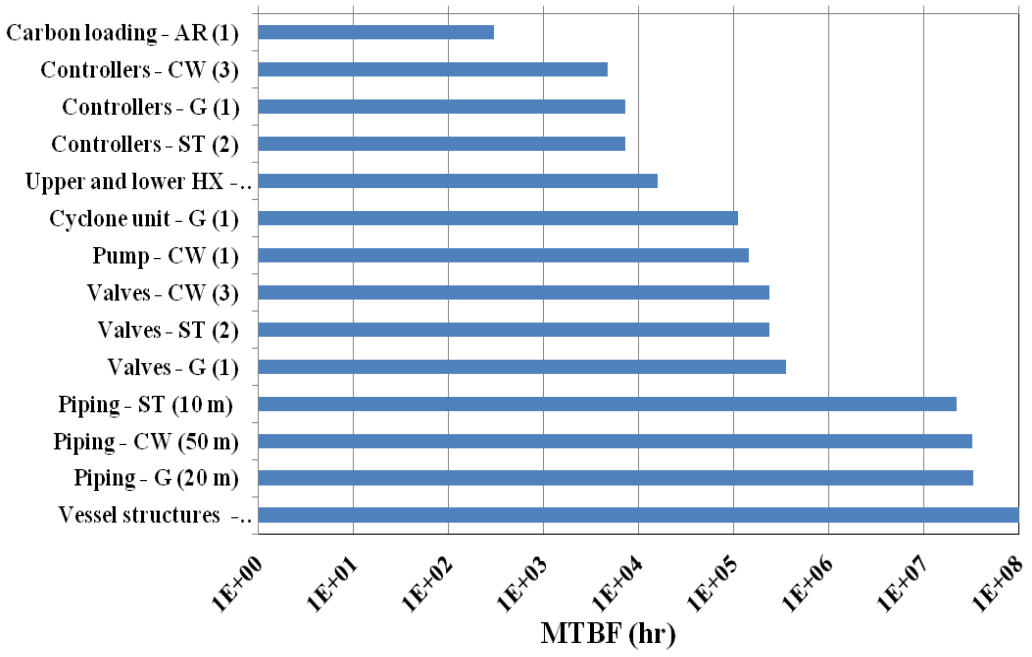

Figure 5 Adsorber Sub-system MTBF 


\begin{tabular}{|l|r|}
\hline Component & Reliability \\
\hline Carbon loading - AR (1) & $2.8 \mathrm{E}-13$ \\
\hline Controllers - CW (3) & 0.16 \\
\hline Controllers - G (1) & 0.29 \\
\hline Controllers - ST (2) & 0.29 \\
\hline Upper and lower HX - AR (1 ea) & 0.57 \\
\hline Cyclone unit - G (1) & 0.92 \\
\hline Pump - CW (1) & 0.94 \\
\hline Valves - CW (3) & 0.96 \\
\hline Valves - ST (2) & 0.96 \\
\hline Valves - G (1) & 0.98 \\
\hline Piping - ST (10 m) & 1.00 \\
\hline Piping - CW (50 m) & 1.00 \\
\hline Piping - G (20 m) & 1.00 \\
\hline Vessel structures - AR (1) & 1.00 \\
\hline
\end{tabular}

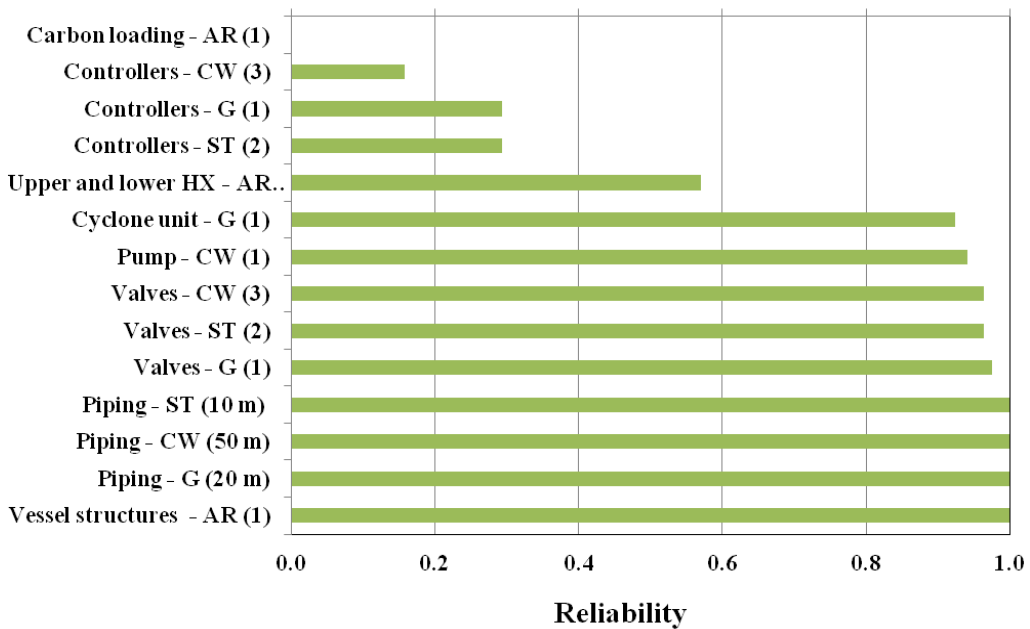

Figure 6 Adsorber Sub-system Reliability

The RMA analysis spreadsheet for the 1-MW hybrid solid sorbent carbon capture system is presented in Table 2. The detailed RMA analysis performed for the adsorber sub-system has been combined with highlevel estimates for the other sub-systems to generate an estimate for overall system reliability. These system roll-ups consider only mechanical-related failures as opposed to phenomena performance-related failures.

Table 2 RMA Analysis Spreadsheet: 1 MW Solid Sorbent Carbon Capture System

\begin{tabular}{|c|c|c|c|c|c|c|c|c|}
\hline \multicolumn{2}{|c|}{ Sub-Systems } & & & & & & & \multirow[b]{2}{*}{8,76} \\
\hline & & & & & \multicolumn{3}{|c|}{ Hours of operation in a year } & \\
\hline & Sub System & $\begin{array}{r}\text { Failure rate } \\
\text { (per MHr) }\end{array}$ & $\begin{array}{c}\text { Failure rate } \\
\text { (per Hr) }\end{array}$ & \begin{tabular}{|c|} 
MTBF \\
(Hr)
\end{tabular} & $\begin{array}{r}\text { MDT } \\
(\mathbf{H r}) \\
\end{array}$ & $\begin{array}{c}\text { Probability } \\
\text { of UnA }\end{array}$ & $\begin{array}{c}\text { UnA } \\
(\mathbf{h r} / \mathbf{y r})\end{array}$ & $\begin{array}{c}\text { Reliability } \\
\text { (annualized) }\end{array}$ \\
\hline 1 & Flue Gas Prep & 500 & $5.0 \mathrm{E}-04$ & 2,000 & 120 & 0.06 & 496 & $1.3 \mathrm{E}-02$ \\
\hline 2 & Adsorber* & 581 & $5.8 \mathrm{E}-04$ & 1,721 & 130 & 0.07 & 615 & $6.2 \mathrm{E}-03$ \\
\hline 3 & Sorbent Transport & 400 & $4.0 \mathrm{E}-04$ & 2,500 & 150 & 0.06 & 496 & $3.0 \mathrm{E}-02$ \\
\hline 4 & Regenerator** & 2,500 & $2.5 \mathrm{E}-03$ & 400 & 212 & 0.35 & 3,035 & $3.1 \mathrm{E}-10$ \\
\hline 5 & $\mathrm{CO}_{2}$ Prep & 396 & 4.0E-04 & 2,525 & 33 & 0.01 & 113 & $3.1 \mathrm{E}-02$ \\
\hline & Total & 4,377 & 0 & 228 & 169 & 0.42 & 3,721 & $2.2 \mathrm{E}-17$ \\
\hline & * without adsorptio & n failure & & & & & & \\
\hline & $* *$ without desorpti & ion failure & & & & & & \\
\hline
\end{tabular}

The sub-systems have been rank ordered to demonstrate the critical contributors to risk for the carbon capture system as shown in Figure 7. Since the results are all roughly the same order of magnitude, the tornado diagram has been presented on a linear scale. The estimates for MTBF and Reliability are similarly presented in Figure 8 and Figure 9, with MTBF on a linear scale and reliability on a log scale, due to the regenerator being many orders of magnitude less reliable than the other sub-systems. Again, the figures may suggest disagreements between MTBF and UnA with regard to the ranking of critical risk components. This difference is attributed to the definition of UnA, which accounts for the down time to repair; therefore, in cases where systems have a relatively high failure rate but lower MDT, the failure rate will not be as critical as similar failure rates with longer repair times. 


\begin{tabular}{|l|r|}
\hline Sub-System & $\begin{array}{c}\text { Corrected } \\
\text { UnA } \\
\text { (hr/yr) }\end{array}$ \\
\hline Regenerator** & 3035 \\
\hline Adsorber* & 615 \\
\hline Sorbent Transport & 496 \\
\hline Flue Gas Prep & 496 \\
\hline $\mathrm{CO}_{2}$ Prep & 113 \\
\hline
\end{tabular}

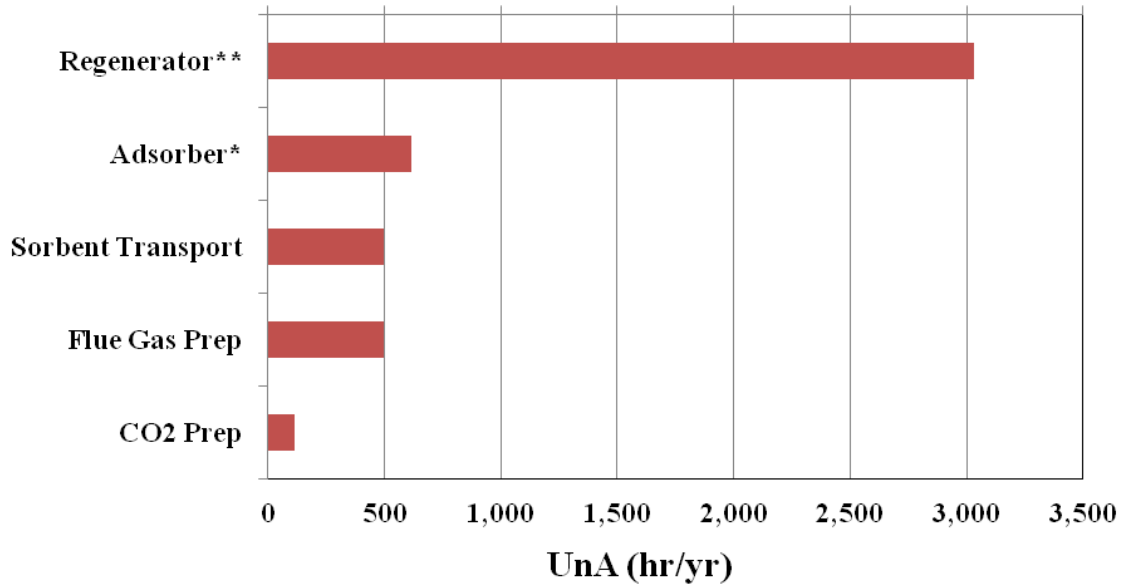

* without adsorption failure

** without desorption failure

\section{UnA (hr/yr)}

Figure 71 MW Hybrid Solid Sorbent Carbon Capture System Unavailability

\begin{tabular}{|l|r|}
\hline Sub-System & $\begin{array}{c}\text { MTBF } \\
\text { (hr) }\end{array}$ \\
\hline Regenerator** & 400 \\
\hline Adsorber* & 1721 \\
\hline Flue Gas Prep & 2000 \\
\hline Sorbent Transport & 2500 \\
\hline $\mathrm{CO}_{2}$ Prep & 2525 \\
\hline \multicolumn{2}{|l|}{} \\
\hline * without adsorption failure \\
\hline$* *$ without desorption failure \\
\hline
\end{tabular}

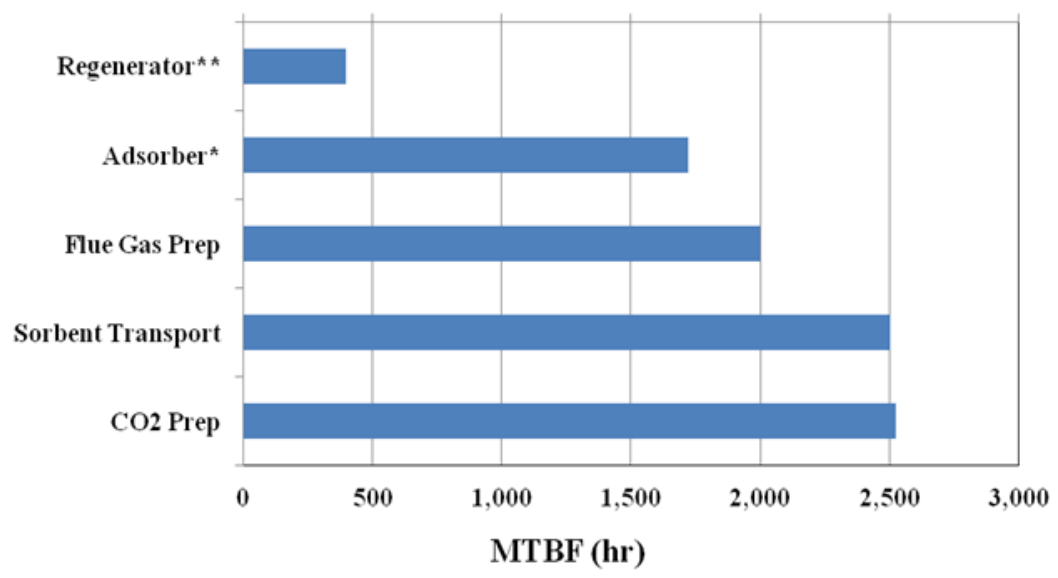

Figure 81 MW Hybrid Solid Sorbent Carbon Capture System MTBF

\begin{tabular}{|l|r|}
\hline Sub-System & $\begin{array}{c}\text { Reliability } \\
\text { (annualized) }\end{array}$ \\
\hline Regenerator** & $3.1 \mathrm{E}-10$ \\
\hline Adsorber* & $6.2 \mathrm{E}-03$ \\
\hline Flue Gas Prep & $1.3 \mathrm{E}-02$ \\
\hline Sorbent Transport & $3.0 \mathrm{E}-02$ \\
\hline $\mathrm{CO}_{2}$ Prep & $3.1 \mathrm{E}-02$ \\
\hline
\end{tabular}

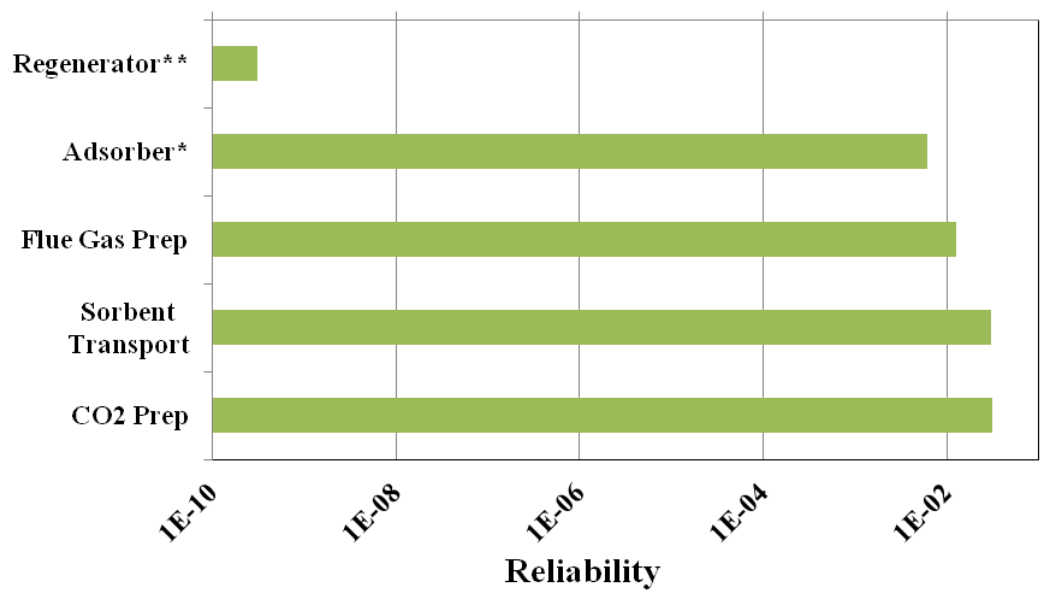

Figure 91 MW Hybrid Solid Sorbent Carbon Capture System Reliability 


\subsection{Discussion}

The objective of the risk analysis activities is to utilize the Risk Analysis Team's computational tools, methods, and capabilities to estimate the probability of failure to meet the targeted carbon capture system performance goal (90\% capture efficiency). Research on probabilistic risk analysis and our initial exploration poignantly highlight the multi-faceted nature of modeling risk contributors to system performance (see Appendix B for a more detailed discussion on carbon capture-related risk contributors, failure modes, and example failure rates). This high level of analytical complexity is especially the case for emerging carbon capture systems.

It should be noted that the intention of the RMA analysis of the 1-MW hybrid, solid sorbent (HSS) putative system design is mainly to illustrate the feasibility of our current conceptual roadmap and approach to risk analysis while utilizing unspecified component characteristics and generic data from standard reliability databases (see Appendix F). Given our simplifying assumptions of constant failure rates and constant MDT with mutual independence among components, the predictions thus generated should be taken as demonstrable or illustrative rather than fully informed or final. Although the estimated reliability of this system is alarmingly low, it should be cautioned that this analysis is an exercise of an evolving methodology and forms a basis upon which the methodology will expand and mitigation strategies will be evaluated. The analysis reflects a low-TRL design with limited knowledge of components, component performance, and tolerance for off nominal operation. Nonetheless, this initial risk analytical exercise provides a basis to consider the relative performance of the HSS components and systems; and to direct further studies and improvements. Our understanding of the system RMA will continue to improve as our model becomes more detailed and as the design matures

Given the state of the design and the high-degree of uncertainty surrounding certain key functions, this analysis has sought to implement the initial phase of our methodology, despite the limited availability of data. Point estimates of component failure rates and down times were selected from industry-standard reliability databases, combined with engineering judgments where needed. These estimates were used to exercise a first order deterministic reliability analysis with discrete relative rankings. The next step in sophistication for the analysis will incorporate appropriate distributions of data in a stochastic analysis, wherein point estimates would have an associated confidence threshold, and generating a spectrum of reliability results. The current results are thus relevant exemplars, but not definitive. The HSS system, as analyzed, shows a very low reliability - equivalent to a system shutdown occurring on average about every 10 days, with the system being unavailable about $40 \%$ of the year. Closer scrutiny suggests that the regenerator subsystem could be the most critical sub-system, driving the low system reliability. The regenerator fails every 2-3 weeks, and is unavailable about one-third of the year. The critical contributors to risk for the regenerator are moving bed solids conveyance, the heat balance system, and steam piping and transport. The heat balance system contains components like a compressor, heat exchangers, pump, and controllers. This regenerator analysis considers the performance of the mechanical components only. The analysis does not include phenomenological issues with respect to the sorbent desorption processes. The physical chemistry may have intrinsic variations or losses as well as perturbations due to changing temperature, pressure, and humidity conditions of the sorbent environments. The impacts of interactions between the sorbent and the mechanical structures are also ignored. Performing an initial heuristic estimation of the RMA of the adsorption process in the adsorber reactor, it was seen that the reliability of the subsystem may be substantially decreased by off-nominal sorbent behavior.

The next largest, but a far second, driver of low system reliability is the adsorber subsystem, without consideration of carbon loading processes. Considering just the mechanical components, the critical contributors to risk are the ubiquitous flow and level controllers, and the upper and lower heat exchange apparatuses in the adsorber reactor. The controllers have a relatively high failure rate but a short MDT, since they are off-the-shelf items and are easily replaced. The heat exchangers have a lower failure rate 
but much longer MDT, since the whole adsorber unit has to be disconnected and opened to repair or replace the heat exchanger tubes or baffles.

For an initial attempt at considering carbon loading processes, a heuristic estimate of the RMA for carbon loading (adsorption) was based on inferences from UQ studies and the TRL uncertainty model (Appendix G). This initial estimate brought focus to the issue of how to consider the "reliability" of a process phenomenon; or even whether that was an appropriate means to use, as compared, say, to the subsystem being considered as out of nominal balance or in degraded performance. If included, the carbon loading RMA estimates utilized had a substantial impact on reducing the subsystem reliability (see Table 1) and topped the risk ranking charts (see Figures 4 - 6).

For the next higher reliability subsystem, the flue gas preparation critical risk contributor was the large motor-driven, centrifugal blower-type compressor component. For the sorbent transport between the adsorber and regenerator, and vice versa, the important component was the solids conveyance device. The solids conveyance device may have a wide variation of RMA values, depending on the manner of conveyance ( e.g. mechanical elevator, screw, or bucket; or a hydraulic or pneumatic driven system).

The risk analysis to date has been deterministic and based on single point estimates of component failure and MDT. As shown by the representative sample of such data in Appendix F, there can be a large variety and range of such estimates (on the order of two to three orders of magnitude). Typically, midpoint estimates were used in this analysis. Large ranges of data exist especially for some of the lower reliability components like solids handling conveyors, compressors, heat exchangers, pumps, and controllers. Beyond the mechanical reliability uncertainties, there is the frontier of process phenomenological risks associated with physical chemistry, process conditions, and process interactions with system components. These uncertainty issues argue the need for better system and component specifications; and for stochastic risk studies utilizing probabilistic distributions of performance informed by UQ studies, TRL uncertainty factors, and process simulations. Such efforts should reveal an informative spectrum of results, which can aid the choice of system design tradeoffs.

Despite the state of the design, this preliminary analysis highlights the role that component and phenomena performance uncertainty plays in overall system risk. The results of this early analysis highlight the key research areas where process understanding improvement is needed. Examples include the adsorption and desorption characteristics in the adsorber and regenerator, respectively; interactions of the sorbent solids with both passive and active mechanical structures; controllability of flows, and of temperature, pressure, and humidity profiles; and accessibility and maintainability of components and sorbent management.

In practice, for systems of this complexity, a number of mitigation strategies might be implemented to bring unavailability into a tolerable range. Some strategies, such as component-specific performance data are used to make the model more accurate, although this strategy requires a high TRL for usefulness. Another strategy is to place higher restrictions on the procurement process such that higher reliability components are specified and purchased for the system, resulting in a higher reliability system in fact as well as modeling, although again a high-TRL strategy. At this low-TRL stage in the design, the mitigation strategies having the greatest impact have to do with design changes, such as creating redundancy in the design such that a failed component can be repaired while the in-line spare is being operated, eliminating the need to shut down because of the component failure. This redundancy with repair may result in a higher incidence of component failure overall, but with a marked decrease in unavailability. 


\subsection{Future Work}

This report has presented our preliminary results for technical risk modeling of the $1 \mathrm{MW}$ hybrid solid sorbent carbon capture system. The initial effort in applying risk analysis to the $1 \mathrm{MW}$ system showed promise for further development of some of the approaches, methods, and techniques, which were adapted from standard probabilistic risk analyses (PRA) practices. The analysis approach used a system engineering functional analysis to identify the key system features and elements pertinent to impacting the high-level performance criteria (carbon capture efficiency) through the operational availability (or unavailability) of the system functions. The risk measure utilized in this study to characterize the risk was the "unavailability". Two dimensions to this risk measure were considered: failure rate (as the likelihood) and mean downtime (MDT) (as the consequence). The failure rate depends on measured failure data from databases for off-the-shelf generic devices when available or estimated using engineering or expert assessments when not available. Thus, the failure rate data may be discrete point estimates or a range of values or a distribution. The MDT includes estimates for repair time as well as other logistical delays. Since the hybrid system is at a very low TRL, we ended up using notional components in certain cases.

In the next project phase, additional endeavor will focus on continuously enhancing and transforming the current deterministic risk analysis modeling approach with simplified assumptions into a more probabilistically-oriented analytical approach. As discussed in the methodology section, the current effort has focused on estimating functional failure and reliability as a launching point for more comprehensive risk analysis in the near future. The RMA analysis spreadsheet, based on point estimates of mutually independent and constant failure rates and MDTs of components, was used to determine discrete relative rankings of RMA metrics. These failure rates and MDTs were selected or sampled from a range or distribution of possible values for each component considered. The next step is to use these distributions of data for each component (including the sorbent adsorption and desorption) in a stochastic rather than deterministic analysis engine. This stochastic analysis will use Monte Carlo-based techniques to sample reliability data for each component and to combine such data into calculating the system reliability as described by the RBD. Thus, there will be a spectrum or distribution of reliability results (based on the extent of Monte Carlo simulations) for the system RBD. This approach will provide a more comprehensive measure of the probability of the system being in a non-failure state over some time period. Sensitivity studies on such reliability distributions should provide additional insights into the critical contributors to risk as well as potential statistical correlations or couplings among system components.

Beyond this development stage, the probabilistic approach to performance risk - the probability that the system or unit fails to meet a specified minimum performance requirement at some point in timeespouses a systemic perspective. This approach tends to utilize logic techniques such as fault trees or decision trees to create a scenario of plausible combinations of events with an impact on a specified topevent or scenario endpoint (e.g., the adsorber captures $90 \%$ of the $\mathrm{CO}_{2} 85 \%$ of the time) ${ }^{1}$. By using uncertainty-laden likelihood and consequence distributions for each contributor to the scenario, and then multiple Monte Carlo simulations or realizations of the scenario, the endpoint event probability of performance failure or success can be modeled stochastically. Such a systemic approach holds the promise of being able to incorporate degraded component performance data and time-dependent characteristics in advanced analyses. Thus, this probabilistic approach offers risk information at a finer granularity, and produces a risk-informed view of the system. The stochastic approach will also provide better coupling between the component-related and the phenomena-related portions of system failure or degraded performance.

\footnotetext{
${ }^{1}$ For instance, such events might include: change in flue gas composition; failure of passive mechanical parts; insufficient cooling water; retarded sorbent flow; sorbent degradation; loss of humidity control, etc.
} 
In final summary, a detailed reliability analysis of one of the large functional blocks (adsorber subsystem) has been completed. In future work, one immediate task is to flesh out the four remaining highlevel functional blocks. Once complete, the analysis will form the baseline against which follow-up analyses will be compared. In addition, for the upcoming technical risk product release, the RMA analysis spreadsheet will be modified to allow user input. The spreadsheet will later be modified to incorporate stochastic treatment of mechanical- and phenomena-related failure/degradation into a higher order confidence on key system performance metrics. Future work will also include evaluation of mitigation strategies for system risk reduction.

\section{References}

Adams, J. Risk. London, UK: Rutledge, 1995.

Alexander, C. Value-at-Risk Models. West Sussex, UK: Wiley, 2009.

Balci, O, Sargent, RG. A methodology for cost-risk analysis in the statistical validation of simulation models. Communications of the ACM, 1981; 24(4):190-197.

Browning, TR, Eppinger, SD. Modeling impacts of process architecture on cost and schedule risk in product development. IEEE Transactions on Engineering Management, 2002; 49(4):428-442.

Chapman, BC. A risk engineering approach to project risk management. International Journal of Project Management, 1990; 8(1):5-16.

Ciferno, JP, Fout, TE, Jones, AP, Murphy, JT. Capturing carbon from existing coal-fired power plants. Chemical Engineering Progress, 2009; 105(4):33-41.

Dale, CB, Letellier, BC. Effects of Insulation Debris on Throttle-Valve Flow Performance: A Subtask of GSI-191 (NUREG/CR-6902, LA-UR-05-7631). Washington, D.C.: Nuclear Regulatory Commission, 2006.

Department of Energy. Basic Research Needs for Carbon Capture: Beyond 2020. Report of the Basic Energy Sciences Workshop for Carbon Capture: Beyond 2020, March 4-5, 2010. Available at http://science.energy.gov/bes/news-and-resources/reports/abstracts/\#Carbon ; retrieved March 8, 2013.

Engel, DW. Initial Decision and Risk Analysis. Richland, WA: Pacific Northwest National Laboratory, 2012.

Hartmann, G, Myer, M. Technical risk, product specifications, and market risk. Pp. 30-43 in Taking Technical Risks: How Innovators, Managers, and Investors Manage Risk in High-Tech Innovations (ed. Branscomb, L and Auerswald, P). Cambridge, MA: MIT Press, 2001.

Hirooka, M. Innovation Dynamism and Economic Growth: A Nonlinear Perspective. Cheltenham, UK: Edward Elgar, 2006.

Hules, KR, Yilmaz, A. From bunker to stack: The Cost-reduction and problem-solving benefits of computational fluid dynamics for utility and industrial power generation. Presented at POWER-GEN International 2002, December 10-12, 2002, Orlando, Florida and Electric Power 2003, March 4-6, 2003, Houston, Texas.

Indian Point 2 and 3. Indian Point Probabilistic Safety Study. Consolidated Edison and New York Power Authority, 1982.

Jones ED, Banks, WW, Altenbach, TJ, Fischer, LE. Relative Risk Analysis in Regulating the Use of RadiationEmitting Medical Devices: A Preliminary Application. Washington, D.C.: NRC Office of Nuclear Material Safety and Safeguards, 1995.

Kaplan, S, Garrick, BJ. On the Quantitative Definition of Risk. Risk Analysis, 1981; 1(1):11-27.

Kincaid CT, Eslinger, PW, Engel, DW, Nichols, WE, Bryce, RW, Bunn, AL. A Stochastic Assessment of Nuclear Waste Management Practices at the Hanford Site, Washington. In Materials Research Society Symposium 
Proceedings (ed. BP McGrail, G.A. Cragnolino). Boston, Massachusetts: Materials Research Society, 2002; 713:275-283.

Klein, JH, Cork, RB. An approach to technical risk assessment. International Journal of Project Management, 1998; 16(6):345-351.

Kwak, YH, Stoddard, J. Project risk management: lessons learned from software development environment. Technovation, 2004; 24(11): 915-920.

Lee, A, Kim, H, Miller, D. Conceptual Design of a 1 MW Pilot Scale Solid Sorbent Carbon Capture Process, CCSI: Carbon Capture Simulation Initiative. Morgantown, WV: National Energy Technology Laboratory, 2010 (a).

Lee, A, Kim, H, Morinelly, J, Eslick, J, Miller, D. NETL ARRA Report on the Development of a Process Design of a Solid Sorbent Carbon Capture Process, CCSI: Carbon Capture Simulation Initiative: Morgantown, WV: National Energy Technology Laboratory, 2012(b).

Lundgren, RE, McMakin, AH. Risk communication: A handbook for communicating environmental, safety, and health risks. Hoboken, NJ: Wiley-IEEE Press, 2011.

Modarres, M. Risk Analysis in Engineering: Techniques, Tools, and Trends. Boca Raton, FL: CRC Press, 2006.

Pawar, R, Bromhal, G, Dilmore, R, Foxall, B, Jones, E, Oldenburg, C, Stauffer, P, Unwin, S, Guthrie, G. Quantification of Risk Profiles for Atmospheres and Groundwater; NRAP-TRS-III-003-2013; NRAP Technical Report Series. Morgantown, WV: U.S. Department of Energy, National Energy Technology Laboratory, 2013.

Pennington, DW, Margni, M, Payet, J, Jolliet, O. Risk and regulatory hazard-based toxicological effect indicators in life-cycle assessment (LCA). Human and Ecological Risk Assessment, 2006; 12(3):450-475.

Rai, V, Victor, DG, Thurber, MC. Carbon capture and storage at scale: Lessons from the growth of analogous energy technologies. Energy Policy, 2009; 38: 4089-4098.

Renn, O. Concepts of Risk: A Classification. Pp. 53-79 in Social Theories of Risk (S, Krimsky and D. Golding, eds) Westport, CT: Praeger, 1992.

Scott MJ, Brandt, CA, Bunn, AL Engel, DW, Eslinger, PW, Miley, TB, Napier, BA, Prendergast-Kennedy, EL, Nieves, LA. Modeling long-term risk to environmental and human systems at the Hanford Nuclear Reservation: Scope and findings from the initial model. Environmental Management, 2005; 35(1):84-98.

Smith, PG, Merritt, GM. Proactive Risk Management: Controlling Uncertainty in Product Development. New York, NY: Productivity Press, 2002.

Wildavsky, A, Dake, K. Theories of Risk Perception: Who Fears What and Why? Daedalus, 1990; 119(4): 41-60

Wu, DD, Kefan, X, Gang, C, Ping, G. A risk analysis model in concurrent engineering product development. Risk Analysis, 2010; 30(9):1440-1453.

Xu, Z, Jinno, K, Kawamura, A, Takesaki, S, Ito, K. Performance risk analysis for Fukuoka water supply system. Water Resources Management, 1998; 12(1):13-30.

Zio, E. An Introduction to the Basics of Reliability and Risk Analysis. Singapore: World Scientific Publishing Company, 2007. 


\section{Appendices}

\section{Appendix A: Review of Carbon Capture System Components and Functions}

The conceptual design for the one MW carbon capture system comprises two main systems: a two-stage fluidized adsorber bed and a moving bed regenerator ( see Figure A.1). ${ }^{2}$ As defined in this report, the process flow begins with introduction of flue gas consisting of carbon dioxide, water, and nitrogen, and ends with compression of $\mathrm{CO}_{2}$ for pipeline transport. The system can be further decomposed into six major functions:

1. Preparation of flue gas

2. Carbon dioxide adsorption

3. Loaded sorbent transport to regenerator and pre-heating of sorbent

4. Sorbent regeneration

5. Regenerated sorbent transport to adsorption unit

6. Carbon dioxide recovery

The following description of the process is based on the NETL American Recovery and Reinvestment Act (ARRA) Milestone Report. ${ }^{3}$

\section{(1) Preparation of flue gas}

The flue gas stream, Figure A.1, coming from a coal power plant, see diamond " 1 " on left side of figure, is assumed to be comprised of carbon dioxide $\left(\mathrm{CO}_{2}\right)$, water $\left(\mathrm{H}_{2} \mathrm{O}\right)$, and nitrogen $\left(\mathrm{N}_{2}\right)$. The flue gas stream is compressed, CPR-001, to meet conditions required for the feed before being introduced into the adsorber, ADS-001. The compressed flue gas stream, 2, is cooled with cooling water, 3, in the heat exchanger, GHX-001, where condensate, 6, which comprises condensed water vapor, may be removed. The cooled flue gas stream, 4, passes into the adsorber, ADS-001, through a gas distributor. The major system components in this step are the compressor and the heat exchanger. The heat exchanger requires cooling water from the plant. Process considerations for the risk assessment that need to be considered include the variability of the incoming gas stream in the three main components $\left(\mathrm{H}_{2} \mathrm{O}, \mathrm{N}_{2}\right.$ and $\left.\mathrm{CO}_{2}\right)$ as well as the presence of impurities in the flue gas that could negatively impact the adsorption capability of the solid sorbent.

\footnotetext{
${ }^{2}$ Lee, A, Kim, H, Morinelly, J, Eslick, J, Miller, D. NETL ARRA Report on the Development of a Process Design of a Solid Sorbent Carbon Capture Process, CCSI: Carbon Capture Simulation Initiative. Morgantown, WV: National Energy Technology Laboratory, 2012(a)

${ }^{3}$ Lee, A, Kim, H, Miller, D. Conceptual Design of a 1 MW Pilot Scale Solid Sorbent Carbon Capture Process, CCSI: Carbon Capture Simulation Initiative. Morgantown, WV: National Energy Technology Laboratory, 2012(b).
} 


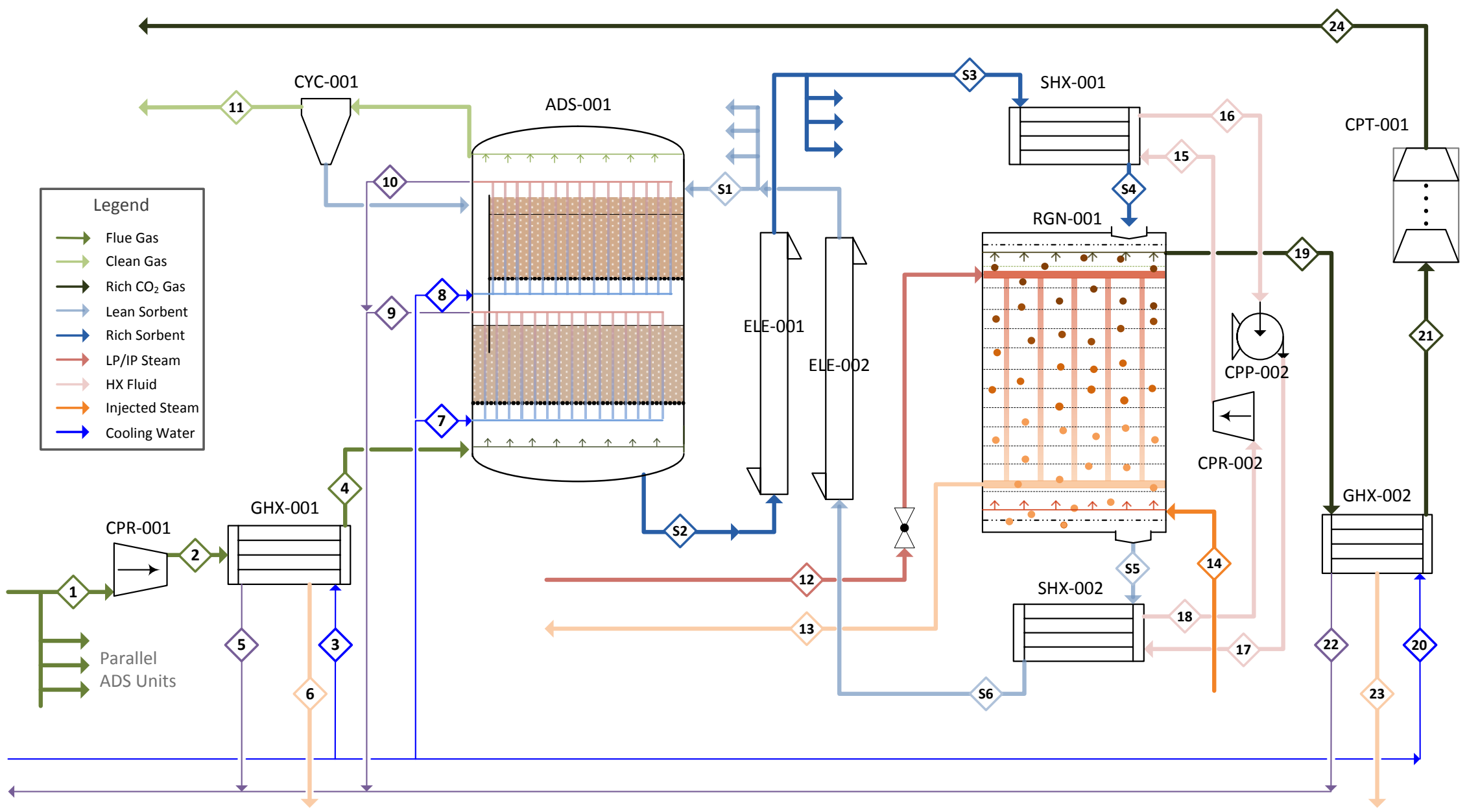

Figure A.1 Process Flow Diagram (Lee et al. 2012, p. 16) 


\section{(2) Carbon dioxide adsorption}

The fresh sorbent stream, $\mathrm{S} 1$, is introduced to the top of ADS-001 and adsorbs $\mathrm{CO}_{2}$ and $\mathrm{H}_{2} \mathrm{O}$ from the flue gas, yielding the loaded sorbent stream, S2. The flow rate of fresh sorbent for each adsorber is calculated by dividing total regenerated sorbent flow rate by the number of adsorber units. In the adsorber, sorbent is cooled to remove heat generated by the exothermic adsorption reaction. This cooling is obtained via thermal contact with water-cooled heat exchange tubes. The $\mathrm{CO}_{2}$-depleted gas stream, 11 , produced from gas-solid contacting, is sent to stack via the cyclone unit, CYC-001, which is used to capture any small sorbent fines entrained in the $\mathrm{CO}_{2}$-depleted gas stream and return them to the adsorber unit. The heat exchanger requires cooling from the plant. Within the adsorber unit, some of the issues of concern from a process viewpoint are the temperature control of the system to maintain ideal binding conditions, adequate interaction of the flue gas with the beads, and proper humidity control for optimal binding.

\section{(3) Loaded sorbent transport to regenerator and pre-heating of sorbent}

The loaded sorbent stream is conveyed by a solid moving system, ELE-001, e.g., a bucket elevator, pneumatic conveyor, or some other means, to a heat exchanger, SHX-001, for pre-heating prior to introduction into the regenerator. This heat exchanger is used in connection with another heat exchanger, SHX-002, on the sorbent outlet side of the regenerator. The sorbent is pre-heated by heat-exchanging with recovered heat from hot sorbent at the bottom of regenerator, RGN-001. The sorbent stream for each unit, S3, is partially heated via thermal contact with heat-exchange surface in the SHX-001. The opposite side of SHX-001 is in contact with saturated steam, 15, which has been vaporized from saturated liquid, 17, by the hot adsorbent via thermal contact with heat-exchange surface in the heat exchanger, SHX-002, and which is circulated between the two heat exchangers by small compressor, CPR-002, and pump, CPP-002. The main components are the solid moving system and the heat exchanger that depends on a compressor and a pump. Proper pre-heating of the loaded sorbent depends on the appropriate balancing of the heat exchangers used for sorbent temperature control before and after the regenerator.

\section{(4) Sorbent regeneration}

In the regenerator, the pre-heated sorbent stream, S4, achieves full heating, corresponding temperature, via thermal contact with in-reactor heat exchanging tubes. In the tube side of RGN-001, hot steam, 12, extracted at the Low Pressure/Intermediate Pressure (LP/IP) crossover in the power plant turbines is used as a heat source. The effect of heating causes adsorbed species to be desorbed, yielding a regenerated sorbent. In addition, a mild condition steam, 14, is also injected at the bottom of reactor to enhance desorption of $\mathrm{CO}_{2}$ by reducing the mole fraction of $\mathrm{CO}_{2}$. This process depends on hot steam and mild condition steam from the plant. For regeneration of the solid sorbent, the temperature and humidity levels within the regenerator need to be controlled to minimize degradation to the sorbent, minimize energy utilization, and maximize $\mathrm{CO}_{2}$ release from the sorbent.

\section{(5) Regenerated sorbent transport to adsorption unit}

The hot regenerated sorbent, S5, leaving the bottom of RGN-001, is partially cooled in heat exchanger, SHX-002, and is delivered again to the top of the adsorber by a solid moving system, ELE-002. As described previously, the heat exchanger, SHX-002, is used in conjunction with heat exchanger SHX001 to maintain the proper temperature of the sorbent before and after the regenerator.

\section{(6) Carbon dioxide recovery}

The injected steam and desorbed species are drawn through the gas collector in stream, 19, at the top of RGN-001 and pass through a water-cooled heat exchanger, GHX-002, to condense water vapor, 23, before being carried into the compression train, $\mathrm{CPT}-001$. The $\mathrm{CO}_{2}$-rich product gas is compressed for pipeline transportation. The heat exchanger depends upon cooling water from the plant. Throughout the process, there is the concern of physical degradation of the sorbent to the point that it can no longer be utilized in the process. 
PNNL-22563

\section{Appendix B: Definition of Performance Criteria and Risk Measures}

The overarching goal of the carbon capture program is to capture $90 \%$ of the $\mathrm{CO}_{2}$ emissions from postcombustion processing of flue gas from new and existing coal-fired power plants with less than a $30 \%$ increase in the cost of electricity. To help accomplish that goal, the carbon capture risk analysis team aims to develop a robust risk analysis methodology and tools to enable users to make informed technology investment decisions.

The carbon capture risk analyses depend on the functional performance of the carbon capture system design and operational processes. During the operational lifetime of the plant, there can be variations in component behaviors, leading to the failure to meet the expected system performance. This system shortfall could be caused by components that fail to meet their specified operating performance, or failure of component integration, which negatively affects productivity. Such events are vulnerabilities or risks that can adversely affect the carbon capture plants, and raise financial, technical, and regulatory concerns to decision makers. Managing these risks may be achieved through risk mitigation practices, which, for example, enhance the reliability of the design or implement resilient operational and maintenance practices to sustain the performance requirements of the carbon capture system. In risk analysis, reliability, maintainability and availability are arguably the most often employed performance criteria and risk measures. We define and discuss the below.

Reliability: Reliability is the probability that an item will perform its intended function over a desired time period, without failure, under specified conditions, with a desired confidence. Describing a complex system in terms of its reliability alone is not, however, sufficient to characterize the system's performance. Reliability needs to be assessed in consideration of both availability and cost of ownership (maintainability).

Availability: Availability is defined as the probability that a system will be operating properly during the times that it is scheduled to operate. Its complement, unavailability, is often referred to as the probability of failure to operate on demand. Unlike reliability, availability accounts for the time to repair a failed item and return to operation. Specifically, Operational Availability estimates include all of the down time associated with scheduled and unscheduled maintenance, such as waiting for access, identifying repair, time for accessing spare parts and/or maintenance personnel, as well as time for repairing and returning component (system) to service.

Maintainability: Maintainability includes actions implemented to restore a failed item to full function as well as preventative maintenance and sparing strategies which serve to prevent item failure from impacting system performance.

For quantitative analysis, reliability, also known as probability of survival, is often measured in terms of its inverse, failure rate. A component's anticipated failure rate is a function of the nature of the anticipated failure. Some component failures reflect inherent characteristics of the component (e.g., manufacturing quality and age) while some random failures are independent of the component operating life. The familiar bathtub "Observed Failure Rate" (blue line) curve (Figure B.1) aggregates the characteristics of initially high rates of early component failure due to manufacturing defects (red line), the near constant failure rate of the primary useful component operating life (green line), and the increasing failure rate as wear-out issues become dominant (yellow line). ${ }^{1}$ In a broad sense, early component failures are managed under procurement quality control, the wear-out phase failures are managed under maintenance, and random failures provide the primary basis for reliability analyses that feed back into system design.

\footnotetext{
${ }^{1}$ The bathtub curve diagram can be retried from http://en.wikipedia.org/wiki/Bathtub_curve.
} 
PNNL-22563

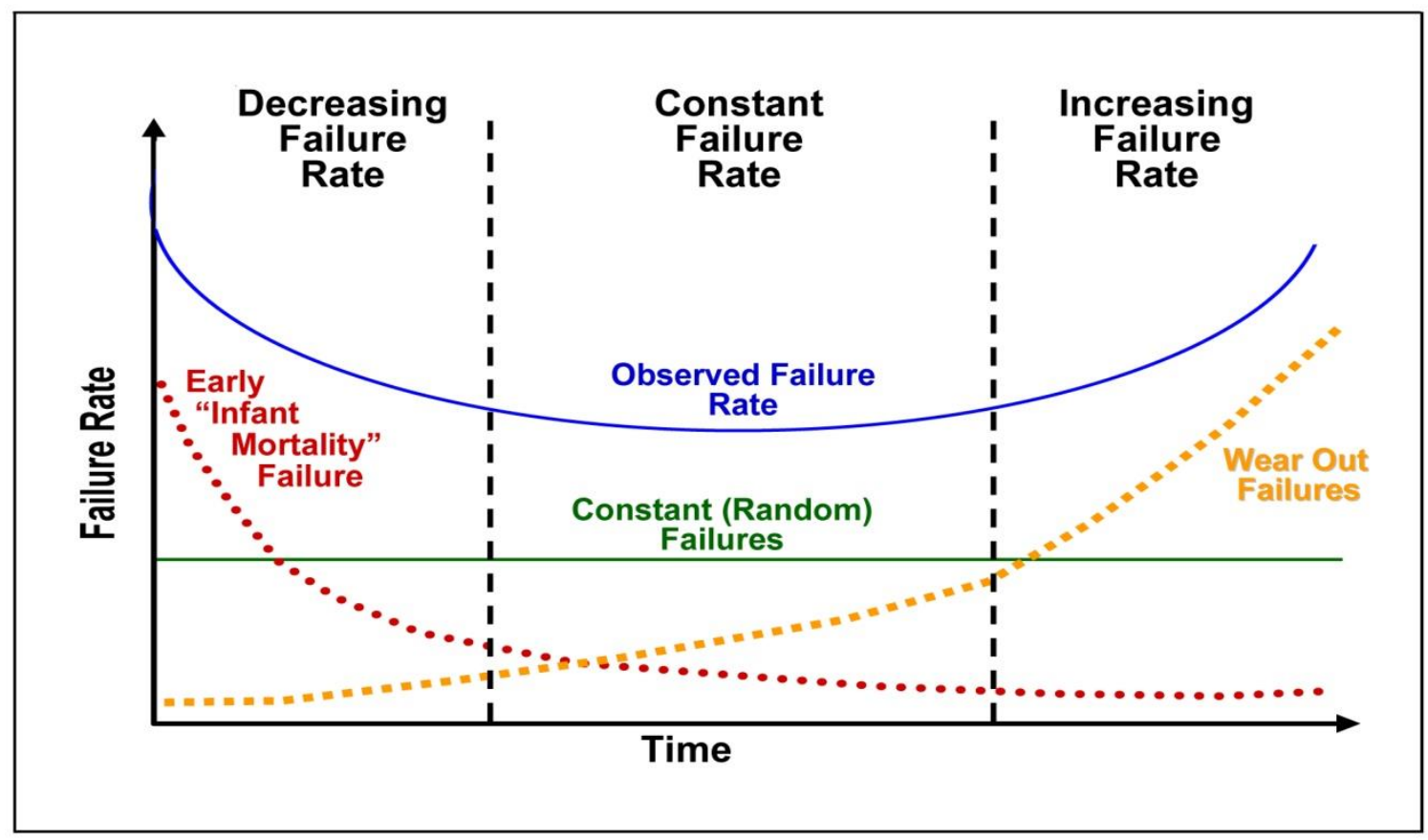

Figure B.1 Component Failure Rate over Time

Considering the constant failure rate region of equipment life and ignoring infant mortality and wear-out (aging) failures, the corresponding reliability of an individual component is exponential,

$$
\text { Reliability }=\mathrm{e}^{-\lambda t}
$$

where $\lambda$ is the constant failure rate. Typically, a constant restoration time is assumed in the estimation of the time required to return the component (system) to operational status after a failure. Following standard convention, the constant failure rate is identified by $\lambda$ and the constant restoration time by $\tau$. For repairable systems, the inverse of $\lambda,(1 / \lambda)$, is referred to as the Mean time between failure (MTBF). Where all downtime is included, the restoration time, $\tau$, is referred to as the Mean down time (MDT). Using these symbolic representations we may express Operational Availability, $A_{o}$ as:

$$
A_{o}=M T B F /(M T B F+M D T)
$$

An estimate of operational availability of a system requires an estimate of the MTBF and MDT of the system. In practice, these variables are obtained by summation of component failure and repair rates with consideration of the multiplicity of each equipment type and any redundancy that exists.

MDT can be broken down into two separate increments of time: (1) Meant time to repair (MTTR) is the time necessary to repair a failed system when all the resources (e.g., manpower, spare parts) are available, and (2) Mean logistics delay time (MLDT) is the additional delays caused by the logistics support for the system (for example, the time required to obtain a replacement part, the time awaiting trained personnel, or the time necessary to repair other failed systems). MTTR is a quantification of inherent design in system maintainability, and MLDT is a quantification of system supportability, which includes personnel, repair at other levels, supply support, transportation, and other logistics delays not attributable to actual hands-on maintenance time (i.e., MTTR). These quantifications can be made by a 
host of methods during various phases of development (and with varying degrees of accuracy). Then from Equation 2, the MDT for the system becomes the sum of MTTR and MLDT, and the prediction of the Availability for a system in development is,

$$
A_{o}=\mathrm{MTBF} /(\mathrm{MTBF}+\mathrm{MTTR}+\mathrm{MLDT}) .
$$

Equation 3 can be used to relate the determining elements: reliability (MTBF), maintainability (MTTR), and supportability (MLDT). The use of MTBF to approximate uptime and the use of MTTR and MLDT to approximate downtime provide both the analyst and decision-makers with discrete variables that can be individually managed and modified, regardless of the developmental stage of the system. In the case of continuous operations, where it is assumed that the supportability conditions are met such that MLDT $\sim 0$, then the Availability can be further simplified to:

$$
A_{o}=\mathrm{MTBF} /(\mathrm{MTBF}+\mathrm{MTTR})
$$

where the mean downtime per failure is dominated by MTTR, or MDT $\approx$ MTTR.

For this stage of the technical risk analysis, the appropriate risk measure is the Unavailability, 1 Availability (or $1-A_{o}$ ), of a system performance element. Unavailability describes the proportion of time for which an item is failed and cannot be used. It is the ratio of downtime to total time,

$$
\begin{gathered}
\mathrm{UnA}=(\text { down time }) /(\text { total time })=(\text { down time }) /(\text { up time }+ \text { down time }) \\
\mathrm{UnA}=\mathrm{MDT} /(\mathrm{MTBF}+\mathrm{MDT}),
\end{gathered}
$$

where UnA is the unavailability of the system, MDT is the "mean down time", and MTBF is the "mean (operating) time between (successive) failures". For a constant failure rate $\lambda$; MTBF becomes $1 / \lambda$. Then by multiplying both the numerator and denominator by the constant failure rate $\lambda$, the unavailability of the system becomes

$$
\mathrm{UnA}=\lambda \mathrm{MDT} /(1+\lambda \mathrm{MDT}) .
$$

Since $\lambda$ MDT is usually small $(<0.1)$, then approximately

$$
\mathrm{UnA} \approx \lambda \mathrm{MDT} \text {. }
$$

In this approximation, Unavailability is the same as the probability of failure on demand (PFD). In reliability work, it is usually referred to as unavailability because the word describes the situation of the plant, i.e., not producing. In safety-related work, where a safety function is inhibited, the PFD term is more usual. Thus, for the purposes of the technical risk analysis of the hybrid solid sorbent carbon capture system, the risk measure utilized is Unavailability and risk is operationalized as the product of likelihood and consequence. In this analysis, likelihood is the component failure rate and consequence is the MDT. Thus, Unavailability is the product of the component failure rate and the MDT.

Analytical models for reliability and availability provide estimates of the inherent capability of the design, and can identify the equipment and subsystems that dominate the failure rates as well as the maintenance burden of the system. Analytic models, however, are generally incapable of providing substantial insight into the expected real-world operation of the system. In particular, systems containing complex functional redundancy involving different equipment in the redundant paths are often difficult to model in closed form. Similarly, systems that will operate in a variety of modes, subjecting equipment to varying operational stress levels for differing time periods, cannot be readily examined analytically. Analytical techniques also suffer from the inability to fully handle degraded operating modes. However, modeling of the carbon capture system demands that such modes of operation be examined. This 
modeling is accomplished by employing techniques that allow specific handling of degraded modes of operation. Finally, the impact of logistics constraints, such as the availability and quantity of spare parts and maintenance personnel, is usually beyond the ability of analytical models to fully assess. The problem of needing to examine the expected performance of such complex systems operations according to varying conditions and logistics constraints is solved by the application of open-form solutions such as simulation techniques.

\section{Appendix C: Carbon Capture System Functional Analysis}

Beginning with the conceptual design of the carbon capture system, the process functions are decomposed into simpler, more readily understood subordinate functions. A hierarchy diagram of the process, Figure C.1, is used to better illustrate the individual elements of the process and their relationships. Simplifying on the discussion from Appendix A, the subordinate functions are arranged under five (rather than six) high-level functions:

1. Prepare Flue Gas,

2. Remove $\mathrm{CO}_{2}$ from Flue Gas,

3. Transport Capture Media

4. Regenerate Loaded Capture Media, and

5. Prepare $\mathrm{CO}_{2}$ for Transport. 
PNNL-22563

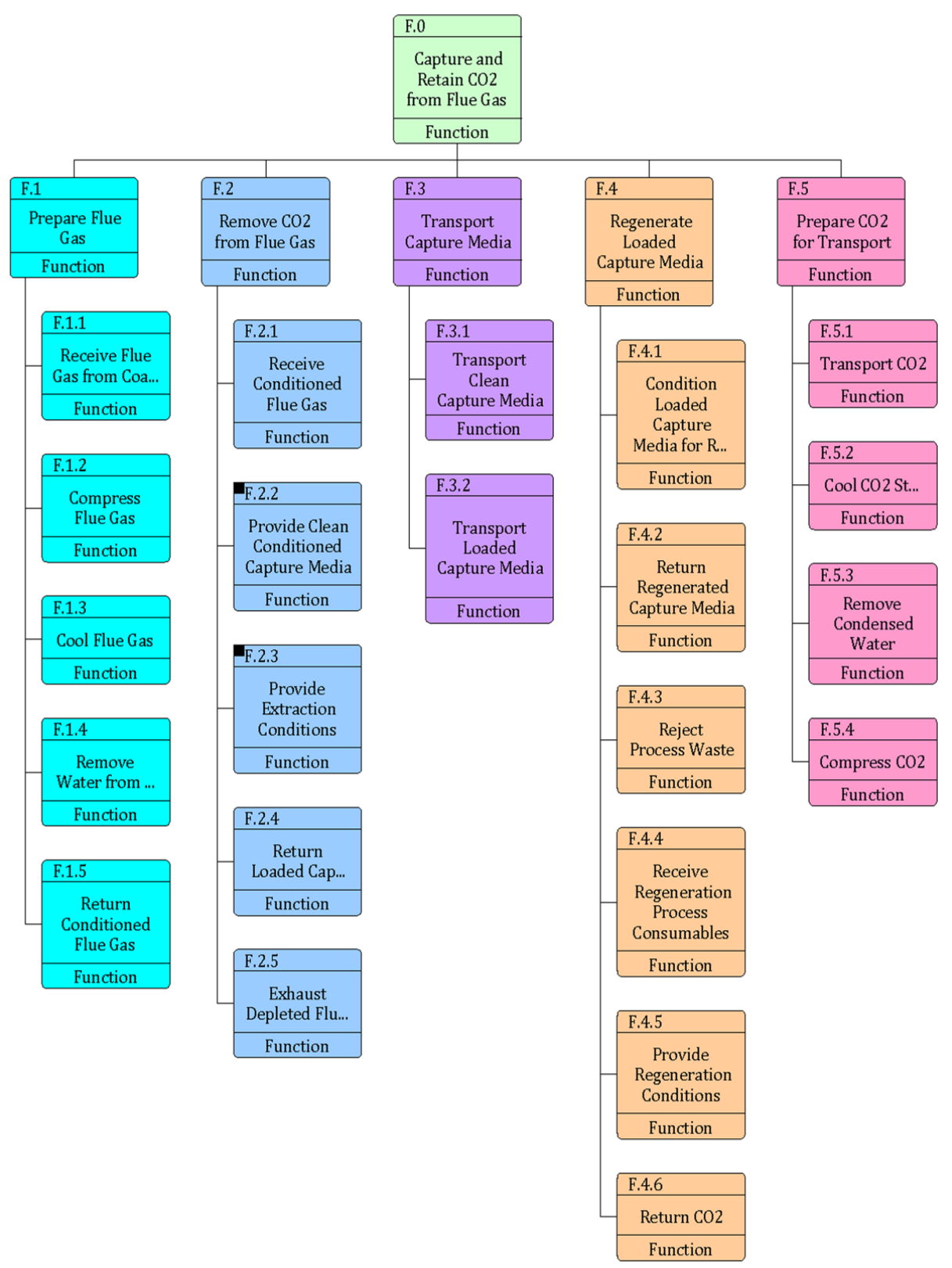

Figure C.1 Functional Hierarchy for Carbon Capture Process

For the sake of analytical parsimony, the functional description shown here has been kept agnostic as to the nature of extraction and regeneration to illustrate the use of systems engineering to capture these high-level functions. As a representation of a fairly generic process design, these functions could be readily translated to the solid sorbent process without having to substantially alter the configuration of the functional design. Each of the high-level functions can be further specified by a high-level functional requirement, which can be refined into discrete performance criteria. As shown in Figure C.2, the function to "Prepare Flue Gas" has the high-level functional requirement to meet the Flue Gas Conditions needed for $\mathrm{CO}_{2}$ extraction. 
PNNL-22563

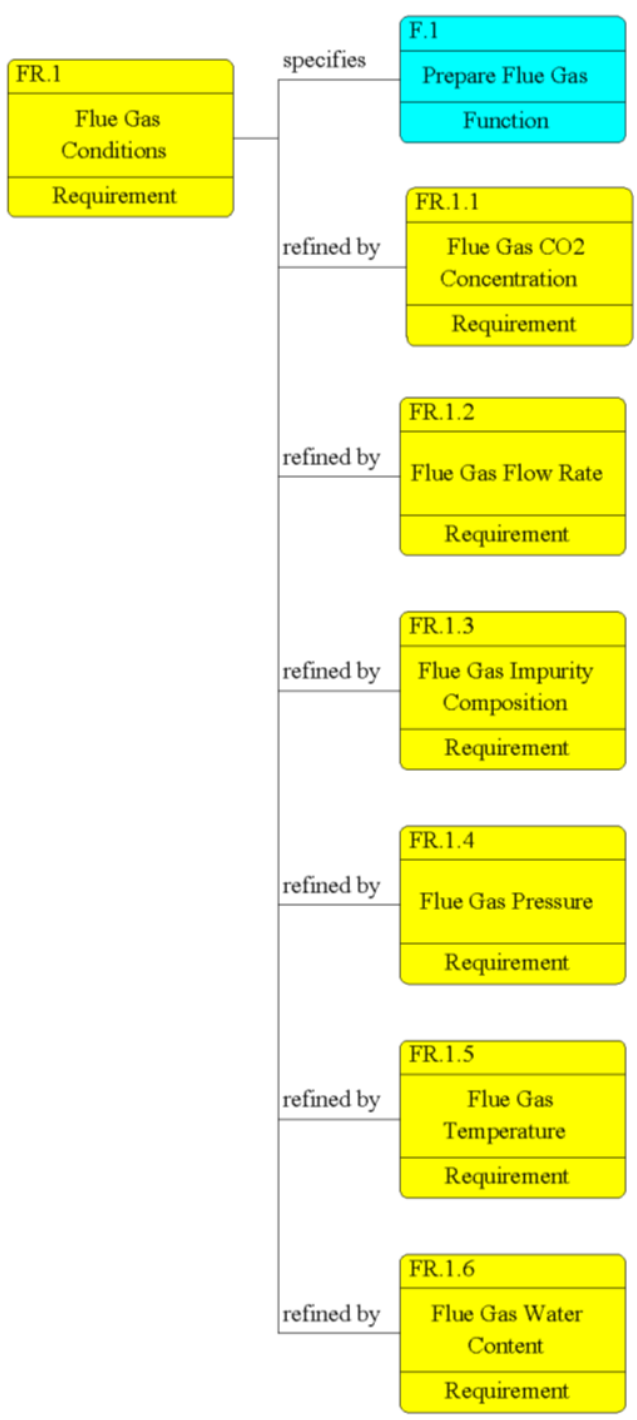

Figure C.2 Key Functional Requirements for Flue Gas Conditions

The fulfillment of the high-level requirement is measured by the specific measurable criteria that define the flue gas conditions to which the process is designed: $\mathrm{CO}_{2}$ concentration, flow rate, impurity composition, pressure, temperature, and water content. By the same token, the primary goal the current risk analysis activity is to is to identify the key functional requirements that must be satisfied for the carbon capture process to meet its performance criteria instead of setting the specific and precise values and tolerances for the parameters for the system under investigation. These requirements, or rather the potential failure to meet these requirements, form the basis of carbon capture technical risk. However, this top-down approach does not address the cause of the failure, but instead identifies the risks introduced by the failure. The example, as shown in Figure C2, reflects early work into the use of functional failure identification of risk as a useful tool, which can be utilized prior to process or component definition. This information is not meant to be inclusive; rather it is intended as an illustration of the technique in its early development. Continued effort will focus on defining the functional requirements and associated risks for the other three high level functions included in Figure C1. 
PNNL-22563

\section{Appendix D: Failure Modes and Effects Identification}

Failure modes and effects analysis (FMEA) is a methodology used to identify potential failure modes, failure causes, failure effects, and problem areas affecting the system or product mission success, hardware and software reliability, maintainability, and safety. FMEA provides a structured process for assessing failure modes and mitigating the effects of the failure modes through corrective actions. When FMEA is performed on hardware in collaboration with the process designer, it is very useful in effecting design improvements. This involves uncovering and resolving, at discrete levels of the system design, single-point failure modes that have an unacceptably high probability of occurrence or a critically severe failure effect. Elimination of single-point failures is one of the primary concerns of performing FMEA. For key findings, design changes should be incorporated to reduce the severity of the failure effects or to minimize the probability of occurrence of the particular failure mode or to increase the detectability of the failure mode in order to reduce risk.

FMEA assesses the effect of each component part failing in possible modes. Here, failure is defined as a component departing from its specifications. The process consists of defining the overall failure modes (usually more than one), and then listing each component fault state that contributes to each failure mode. For identifying the failure modes and hence failure of operational demand, it is essential that the level of performance (LOP) must be defined for each component be defined. Here we focus on the following LOP factors: role, functionality, reliability and availability, survivability, and their interdependence (see detailed definitions and descriptions for these LOP factors, see DNV 2013, p. 17). ${ }^{1}$ In the hybrid solid sorbent study, risk results are presented in terms of the two components of risk: the likelihood of a failure event and its associated consequences. Thus, each event quantified in the risk analysis would correspond to a point in a two-dimensional risk space. Such a representation not only aids in identifying those events or risks of the greatest concerns but also provides information to support decisions about what range of risks (regions of the risk domain) is acceptable.

Risk assessments for complex systems like the carbon capture system must also account for hazards resulting from factors that lie outside of the steady state system design. These other factors include those internal to the facility, such as internal transient events, feedback and control behavior, operations, configuration management, human interaction, and external factors that are not under the control of the facility, such as supply chain logistics, market factors, regulatory changes, and external transient events.

Below we list potential hazards and their causes associated with the presence of $\mathrm{CO}_{2}$ under variable temperature, pressure, and chemical conditions, focusing on threats to meeting the system component performance levels needed. Specific $\mathrm{CO}_{2}$-related threats include material incompatibility or damage, dissolving of lubricants, enforcement walls, internal corrosion, hydrate formation, pressurization resulting from phase and temperature change, boiling liquid evaporating vapor explosion (BLEVE), propagating cracks, and flailing pipes (for more information about the definitions and descriptions of these threat categories, see DNV 2013, pp. 105-109). Other potential hazards are non- $\mathrm{CO}_{2}$ related. Possibly important initiating causes include:

- Dropped objects

- Overpressure from connected pipework or local pump/compressor

- Impact form an adjacent event

- Erosion

- Excessive vibration

- Cyclic loading

${ }^{1}$ Det Norske Veritas. $\mathrm{CO}_{2}$ Riskman Guidance on $\mathrm{CCS} \mathrm{CO}_{2}$ Safety and Environment Major Accident Hazard Risk Management Level 3-Generic Guidance, Report No. I2IJLJW-2, DNV Ltd. Aberdeen, UK, 2013. 
PNNL-22563

- Stress corrosion cracking

- Foundation or support failure

- Material defects

- Fabrication defects

- Rotating equipment failure

- Subsidence

- Environmental impact (e.g., seismic, fire, flood)

- Sabotage or malicious act

Based on the above hazard considerations and the level of process system design available, an evaluation of the hybrid solid sorbent deviations in performance and related failures and effects was carried out. The analysis focused on the performance characteristics of gas and mass flow, subject to desired temperature and pressure profiles, as these are metrics related to carbon loading of the solid sorbent. Deviations from "normal" conditions were considered. A sample of the analysis is summarized below in a hazard identification table (Table D.1). Critical system components, such as pumps and valves, are identified as part of the hazard and failure analysis. Putative modes of failure associated with these components were identified with examples shown in Table D.2. Where possible, the percentage of a component's failure mode was recorded using available databases for generic devices.

Table D.1 Sample Hazard Identification Table

\begin{tabular}{|c|c|c|}
\hline Deviation & Causes & Consequences \\
\hline \multirow{4}{*}{ No flow } & \multirow[b]{2}{*}{ Sorbent buffer tank empty } & Damage to pump \\
\hline & & Loss of fresh sorbent to adsorber vessel \\
\hline & Line frozen & $\begin{array}{l}\text { Loss of sorbent supply to adsorber or } \\
\text { regenerator }\end{array}$ \\
\hline & Valve in line shut & $\begin{array}{l}\text { Possible damage to line as pump dead heads, } \\
\text { i.e., runs against closed discharge line }\end{array}$ \\
\hline More flow & $\begin{array}{l}\text { None (fixed by maximum pump } \\
\text { discharge) }\end{array}$ & \\
\hline Less flow & $\begin{array}{l}\text { Line partially plugged or valve } \\
\text { partially closed }\end{array}$ & $\begin{array}{l}\text { Possible damage to line as pump dead heads } \\
\text { grind against closed discharge line }\end{array}$ \\
\hline \multirow{6}{*}{ Reverse flow } & \multirow{6}{*}{ Pump trips } & Back flow of gas to tank \\
\hline & & Resulting in: \\
\hline & & 1. Possible rupture of tank \\
\hline & & \\
\hline & & $\begin{array}{l}\text { 2. Major release to plant causing potential } \\
\text { toxic cloud and possible vapor cloud explosion } \\
\text { if cloud reaches congested part of the plant }\end{array}$ \\
\hline & & \\
\hline
\end{tabular}


PNNL-22563

\begin{tabular}{|c|c|c|}
\hline $\begin{array}{l}\text { High } \\
\text { temperature }\end{array}$ & $\begin{array}{l}\text { Lack of cooling on the sorbent in } \\
\text { the adsorber unit }\end{array}$ & Possibility of poor adsorber reactor efficiency \\
\hline $\begin{array}{l}\text { Low } \\
\text { temperature }\end{array}$ & Cold conditions & $\begin{array}{l}\text { Possible freezing of lines. Possibility of poor } \\
\text { regenerator efficiency. }\end{array}$ \\
\hline \multirow[b]{2}{*}{$\begin{array}{l}\text { High } \\
\text { pressure }\end{array}$} & Pump dead head & Possibility of overpressure of containments. \\
\hline & $\begin{array}{l}\text { Reverse flow from adsorber or } \\
\text { regenerator units }\end{array}$ & Ditto \\
\hline $\begin{array}{l}\text { Low } \\
\text { pressure }\end{array}$ & None identified & Not seen as a problem \\
\hline
\end{tabular}

Table D.2 Failure Modes and Their Relative Percentage of Occurrence

\begin{tabular}{|c|c|c|}
\hline Item & Mode & Percentage \\
\hline & Failed & \\
\hline Motor & & 65 \\
\hline \multirow[t]{6}{*}{\begin{tabular}{|cl} 
- Brush & \\
- & Commutator \\
- & Lube \\
- & Rotor \\
- Stator &
\end{tabular}} & & $\begin{array}{l}15 \\
10 \\
15 \\
10 \\
15\end{array}$ \\
\hline & Performance & \\
\hline & (degraded) & 35 \\
\hline & - $\quad$ Brush & 15 \\
\hline & $\begin{array}{ll}\text { - } & \text { Commutator } \\
\text { - } & \text { Lube }\end{array}$ & $\begin{array}{l}5 \\
15\end{array}$ \\
\hline & Leak & 50 \\
\hline \multirow[t]{2}{*}{ Pump } & No transmission & 50 \\
\hline & Coil & 10 \\
\hline \multirow[t]{3}{*}{ Relay } & Contact & 90 \\
\hline & Fail to operate & 90 \\
\hline & High leakage & 20 \\
\hline \multirow[t]{4}{*}{ Valve (mechanical) } & External leak & 15 \\
\hline & Passing (internal) & 60 \\
\hline & Sticking & 20 \\
\hline & Fail & 10 \\
\hline Valve actuator & Spurious & 90 \\
\hline
\end{tabular}


PNNL-22563

\section{Appendix E: Candidate Risk Assessment Methods}

\section{Relative Risk Profiling}

Probabilistic risk assessments (PRA) require component failure data to estimate system failure. The traditional PRA process begins with an initial failure event definition and delineates probability and consequence paths that result in risk. The hybrid solid sorbent system is a relatively straightforward mechanical and hardware system with significant process chemistry control issues. It is also a relatively new system and has little operating experience base or data about component performance. Most information resides in the experience base of manufacturers and some users. In addition, the methodology should not be a rule-based but should be a systematic approach to uncovering risk for a range of conditions. For relatively new devices, most of the operating experience data will be qualitative rather than quantitative. Therefore, the risk analysis must not rely only on quantitative data in order to be useful; it should be able to compare a range of data types and data quality. Therefore, an appropriate risk analysis methodology for assessing carbon capture systems must be able to:

- focus on failure modes and performance deviations as primary causes of undesired events;

- provide a flexible framework for performing analyses; and

- be able to incorporate both qualitative and quantitative data.

Among competing methods, relative rankings of risk or risk profiles seem to meet the above criteria. Profile analysis is a general analytic tool applied to the evaluation of both machine failures and process errors in industrial facilities in the past. ${ }^{1}$ It is both a qualitative and quantitative technique for assessing relative risk associated with task or process execution. For the hybrid solid sorbent carbon capture system, the interest is in mechanical failures as well as deviations in the chemical processes. For that purpose, a "task" is defined as a demand for a level of service from a component or a phenomenological process. Hence, "task failure" or a risk event is associated with a component fault or phenomenological deviation, given a demand for that service element.

After the tasks associated with identified hazards and failure scenarios are defined, analysts observe, systematically rate, estimate, or measure the relative likelihood of error as a function of each task, as it is typically performed. After the relative probabilities of task failure (or success) are determined (using past records of incidents or failures) or estimated (using task content experts), the consequence associated with each failure is identified and then rated by magnitude. At this point, the analyst has two estimated or measured relative quantities: the likelihood of failure and the magnitude of consequences. These two point estimates are then multiplied to produce a product reflecting the relative risk associated with each task in a sequence of tasks to be performed.

In the context of carbon capture systems and assuming sufficient quantitative data were available, identification of the highest-risk factors is performed by directly multiplying failure rates by MDT. If quantitative data are substantially lacking, qualitative judgments would have to be used to formalize the rankings on a relative basis. Use of judgment is especially needed for process chemistry phenomena associated with the performance of the adsorber and regenerator reactor vessels. As mentioned above, an advantage of a relative ranking scheme is its ability to compare both qualitative and quantitative data. The best method and data available should be used to estimate a likelihood or measure of consequence

\footnotetext{
${ }^{1}$ Seaver, DA, Stillwell, WG. Procedures for Using Expert Judgment to Estimate Human Error Probabilities in Nuclear Power Plant Operation. NUREG/CR-2743, Decision Science Consortium and Sandia National Laboratories, 1984. See also, Banks, WW. Profile Analysis: An Advanced Analytic Method for Human Error Assessments, EGG-REP-6574, Idaho National Engineering Laboratory, 1984.
} 
for each risk contributor. Although there can be a wide variation in the quality of estimation from risk contributor to contributor, in principle all measures can be compared by means of relative rankings.

To conduct a first-order risk analysis, likely failure rates and consequences for each task were treated as independent from other tasks, and were estimated as if they were independent components. After obtaining data for the primary tasks, the mean values of the failure rate and MDT for each task were used as point estimates of their likelihood and associated consequence. The product of these two numbers then provided a first-order risk estimate for the task. These relative risk profiles thus aid the identification of the high-risk, high-consequence, or critical events, without requiring an absolute quantification of probability, consequence, and risk for each task. While such relative comparisons support determination of the strengths and weaknesses of a particular technology design and its optional strategies, it does not provide the quantification needed to compare different technology options, per se. This comparison would require a much more trusted information base to develop confident quantifications of risk measures that can be directly compared across a common baseline.

\section{Scenario-Based Risk Analyses}

In the first-order risk analysis, likely failure rates and consequences for each task were treated as independent from other tasks, and were estimated as such. However, many faults or consequences may be impacted by other system elements or behaviors. Such effects must be adequately reflected in the risk profiles so that final ranking schemes can incorporate interacting factors.

Scenarios involve plausible concatenations or integration of tasks to validate or adjust the rankings of tasks to ensure appropriate relative rankings. Fault tree and decision tree are common techniques for creating what-if scenarios, which facilitate relative ranking estimation. In either the event tree or fault tree method of defining scenarios, the likelihood and consequence distributions of each contributing event are logically combined (using the probability calculus) to assess the risk of the top or endpoint event. In the case of the first-order risk analysis, the failure rate and MDT data constituted point estimates or discrete distributions. To accomplish the appropriate combinations, the discrete distribution propagation method used in the Zion and Indian Point PRAs can be employed. ${ }^{1}$ Additionally, the Monte Carlo method can be applied to combine distributions, whether they are discrete or continuous, by sampling the associated cumulative distribution functions (CDFs) in a random manner (e.g., Latin Hypercube Sampling). By such means, a probabilistic risk assessment approach is obtained for the evaluation of scenarios and the impacts of task failures.

In general, the MDT of a system or component is an estimated average elapsed time required to perform corrective maintenance, which consists of fault isolation and correction. The repair time of a maintainable unit generally consists of short duration repair periods, a large number of times grouped about some average value, and a small number of long-time repair periods. The former would correspond to the more usual case where the failed unit is replaced by a spare at the operational site on detection of a failure. The long down times would occur when diagnosis is difficult or removing a defective part is complicated. Having a collection of such field data provides the designer an opportunity to assess the MDT of a current system as it matures, or to predict the MDT of a new system according to its features with the current system. An interval estimator for MDT can be developed from the mean of the sample data, within a lower and upper limit with a confidence bound. Unfortunately, an exact MDT of a system or component cannot be found due to data uncertainties.

The distribution most commonly used to describe the actual frequencies of occurrence of system down time is the log normal, because it reflects short duration repair time, a large number of observations

${ }^{1}$ Commonwealth Edison Co. Zion Probabilistic Safety Study. Consolidated Edison Co, 1981. New York State Power Authority, Indian Point Probabilistic Safety Study, 1980. 
closely grouped about some modal value, and long repair-time data points. The MDT distribution for a particular task can be developed by determining the best estimated values of the mean of down times and the variance of those times. Relative probability distributions can be generated along with variance estimates, by developing a frequency distribution of the actual historical data if it is available or of experts' estimates.

The degree of dispersion among expert estimates or different data sources is typically used to produce an estimate of the variance. This point in analysis is where information from uncertainty quantification and other uncertainty considerations can be incorporated. The resulting values then determine the parametric shape of the log normal distribution that represents a range of MDT values. The distributions developed for each risk-pertinent task in the solid sorbent study do not represent true probability distributions in the classical sense. They are based on experiences (of varying degrees) and thus of uncertain probability. Rather, the relative probabilities more accurately represent density functions in the Bayesian sense. In this sense, the attempt was to include all information that is relevant, and such information may be conveyed as a distribution in which height reflects belief and width reflects uncertainty. The large values of uncertainty and the wide variability in the uncertainties from task to task indicate that the first-order risk analysis may not be adequate to represent the combinations of faults among tasks in a risk scenario. Thus, the full fault probability and consequence distributions should be used when estimating risks of scenarios. Failure rate and MDT distributions can be used in Monte Carlo simulations of risk scenarios. In this study, a Monte Carlo method can be used to simulate and evaluate the relative risks of possible fault scenarios. It made full use of the developed failure rate and MDT magnitude distributions and can, in principle, model the interactions among any number of tasks, logically combining distributions. It can be used to aggregate tasks and their distributions, determine best- and worst-case extremes, and perform uncertainty and importance analyses.

Scenarios will be defined through the use of logic diagrams with the tasks as contributing events to the top or endpoint states. For each task, the unit- normalized probability of failure distribution is randomly sampled to select a fault probability. To determine if the fault occurs, a random number is generated to compare to the selected fault probability. If the random number is greater than the fault probability, then the fault is deemed to have occurred. Otherwise, the task fault is ignored. If a fault occurs, the MDT associated with that fault is sampled and recorded. If there are more tasks to include in the scenario then they are similarly sampled. The fault probability, consequence measure, and risk for each task with a fault in this scenario are logically combined with those measures from other failed tasks. This collection of faults and MDTs' constitute a scenario realization. Several realizations are executed to build up distributions of risk, e.g., consisting of the number of scenarios with a specific risk value. The approach to generating distributions associated with simulated scenario realizations is to calculate distributions for the possible combinations of faults and then combine those distributions into the top or endpoint distribution. This approach offered substantial qualitative insights.

In the process of making such calculations, the highest risk scenarios could be identified, as well as those tasks most often contributing to the high-risk scenarios. This identification was subsequently used to indicate which tasks were the most significant contributors to risk. Then, the analyst can select the tasks and their data to be included in a scenario evaluation. The sequential event selection process in the scenario simulation is represented by the "decision tree". For each task, it is decided whether a fault occurs or not based on its probability. If it does not occur, there is no consequence and hence no contribution to risk, and the calculation moves on to the next task and resumes the logic of the decisionmaking heuristic. The endpoints of all the tree's branches correspond to unique outcomes of the scenario, one for each realization of the scenario. These risk realizations can be aggregated into a risk distribution, such as the risk response surface illustrated in the graphic (Figure E.1). Here the vertical axis is the number of realizations of a selected scenario with a given risk value, specified by the outcome's probability and consequence measures, respectively represented by the horizontal axes. All axes values 
are scaled to the maximum value of that axis parameter. A large number of realizations result in a semicontinuous range of probability and consequence parameters and risk values.

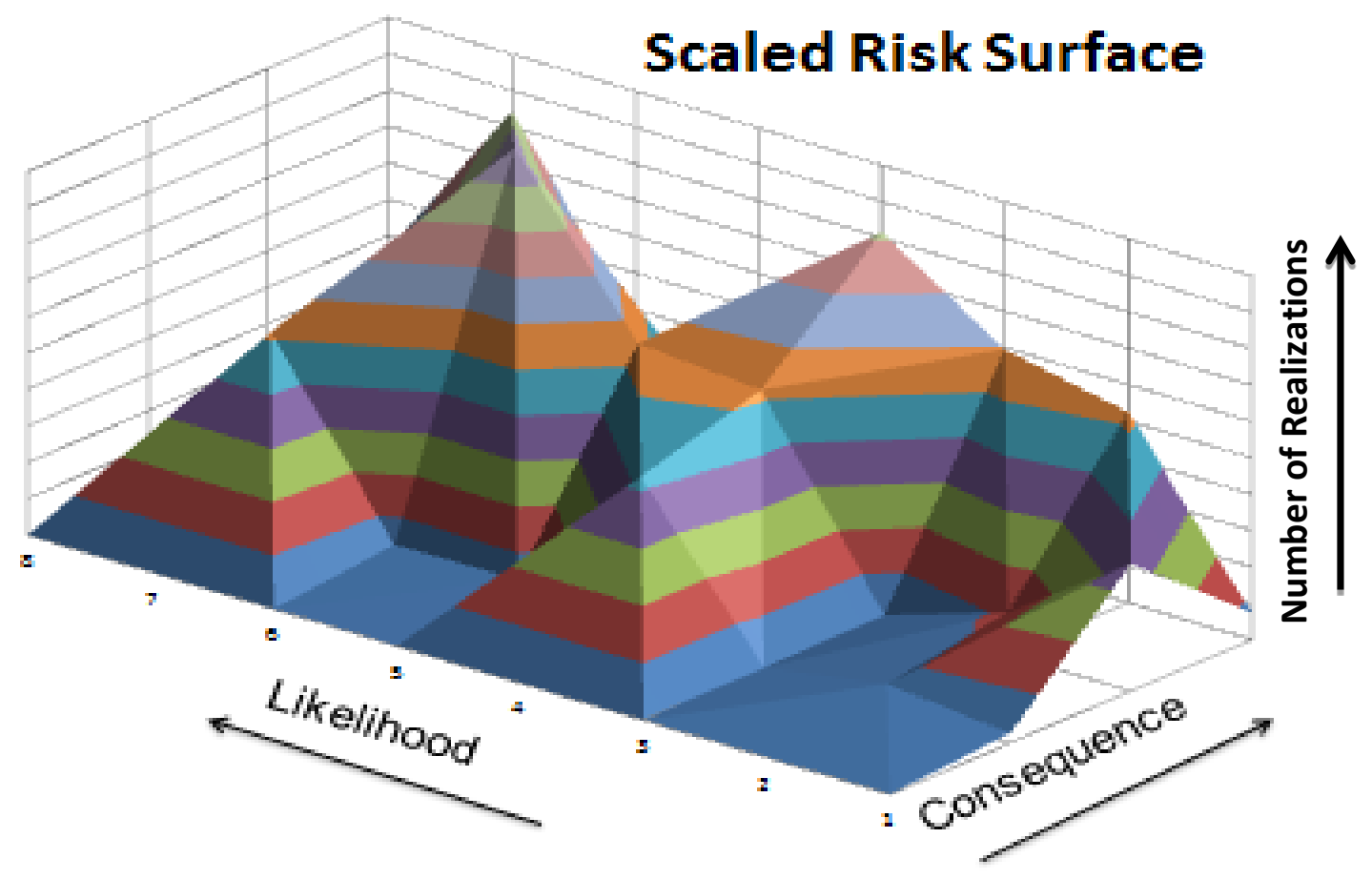

Figure E.1 Representation of the Distribution of the Number of Realizations with a Given Risk Value for a Defined Scenario

Such results can aid in the identification of important risk contributors and factors. The highest risk scenarios can be identified, as well as those tasks most often contributing to the high-risk scenarios. The total fault probability and consequence value for each simulated scenario realization is recorded. Based on the range of these values, probability bins and consequence bins are established to help aggregate the results into forms similar to the risk surface above, where the distribution of the number of realized risk scenarios as a function of the fault probability and consequence is shown. The plot in Figure E. 1 shows a region in risk space associated with the majority of scenario outcomes in the high likelihood, high consequence domain and in the mid-high consequence domain. This graph indicates that, given the data used, there are several scenarios of risk concern, and presumably several opportunities for risk mitigation and future risk reduction.

These results are interesting from a couple of perspectives. First, they indicate prevalent tasks in the higher-risk scenarios. Second, in comparison to the point risk estimates, they show the effects of using the fault distributions rather than just the means. The scenario simulations are anticipated to be used also to perform risk sensitivity studies or examine risk mitigation strategies. This analysis could be performed by modifying the nature of the tasks (components and processes) and hence their failure modes, rates, and consequence distributions. Sensitivity and importance studies can be performed using task distributions to try and identify means to reduce the tasks risks and the high-risk scenarios to which they contribute. The overall impact on system performance of a mitigation strategy can then be tested via scenario simulation results.

\section{Failure Modes and Effects Criticality Analysis (FMECA)}


Similar to FEMA, failure modes, effects, and criticality analysis (FMECA) is a quantitative approach to calculating the criticality of a failure mode. The criticality measure consists of (1) a frequency or likelihood of failure dimension and (2) an impact or consequence measure parameter. FMECA is effective in comparing and ranking failure criticalities and performance thresholds across modes. ${ }^{1}$ Criticality can be used for evaluating and prioritizing risk mitigation investments to address risks during the design stage. With criticality calculations and risk priorities, we can then assess single-point failures that are expressed as likelihood frequencies and impact. The criticality calculation for any single-point failure can be used to support a design-change decision if the risk ranking warrants such an action. A design change may include altering system architecture, and improving system's fault tolerance and redundancy. Below we elaborate on the two components of FMECA: likelihood magnitudes and consequences.

\section{Likelihood Magnitudes}

The objective of the frequency analysis or likelihood of task failure is to examine the events identified, assess the probability/frequency, and identify potential causes. The events to be analyzed are determined by the hazard identification as discussed in Appendix D. The causal analysis of failure events should be appropriate for the context of the risk assessment and should broadly consider factors related to design and operation as well as maintenance with the appropriate and diverse expertise on the risk assessment team (see Footnote 4). Ideally, these requirements are fulfilled with the use of statistical failure data. These basic data are based partly on accident and failure statistics, and partly on detailed cause analysis (e.g. fault-tree analysis). For the current stage of carbon capture $R \& D$, until sufficient relevant statistical failure data are available for $\mathrm{CO}_{2}$ handling systems, caution should be taken when undertaking the frequency analysis. There may be a requirement to undertake more logic tree analysis or other open-form evaluations within the frequency analysis in order to compensate for the relative lack of $\mathrm{CO}_{2}$ relevant statistical failure data.

It should be noted that failure statistics reflect average environment, technologies, and operational standards of the past. Thus, the specific features of the system being assessed and the system's environment should be considered whenever judged necessary. The discussions should compare these specific features to "average conditions" in the relevant area in order to assess whether the system is expected to be exposed to the initiating event more or less frequently than the average. If there are good reasons to expect such differences, the statistical figures should be adjusted accordingly. Table E.1 contains examples of such "averaged" failure rate data for components essential to performance of the hybrid solid sorbent system. Note that the data have a range from low, to average, to high. The data also reflect an assumed and simplified constant failure rate for the component over the considered period of performance of the system.

Table E.1 Example Failure Rates in Million Hours (MHours)

\begin{tabular}{|l|c|c|c|}
\hline \multicolumn{1}{|c|}{ Item } & \multicolumn{3}{c|}{ Failures per MHours } \\
\hline Compressor & & & \\
\hline- Centrifugal, turbine driven & 150 & & \\
\hline - Reciprocating, turbine driven & 500 & & \\
\hline - Electric motor driven & 100 & & 300 \\
\hline Motor (Electrical) & & & \\
\hline - a.c. & 1 & 5 & 20 \\
\hline - d.c. & 5 & 15 & \\
\hline
\end{tabular}

${ }^{1}$ Raheja, DG, Gullo, LJ. (Eds.). Design for Reliability. Hoboken, NJ: Wiley, 2012. 
PNNL-22563

\begin{tabular}{|l|c|c|c|}
\hline- Starter & 4 & & 10 \\
\hline Pressure instruments & & & \\
\hline- Switch & 1 & 5 & 40 \\
\hline- Sensor & 2 & & 10 \\
\hline- Indicator & 1 & 5 & 10 \\
\hline- Controller & 1 & 10 & 30 \\
\hline Pumps & & & \\
\hline- Centrifugal & 10 & 50 & 100 \\
\hline- Electrical & 200 & & 500 \\
\hline Stepper motor & 0.5 & & 5 \\
\hline Temperature instruments & & & \\
\hline- Sensor & 0.2 & & 10 \\
\hline- Switch & 3 & & 20 \\
\hline- Controller & 20 & & 40 \\
\hline $\begin{array}{l}\text { Valves (mechanical, hydraulic, } \\
\text { 25pneumatic, gas (not high temp. } \\
\text { nor corrosive substances)) }\end{array}$ & & & \\
\hline- Ball & & & \\
\hline- Butterfly & 0.2 & 3 & 10 \\
\hline- Diaphragm (single) & 1 & 20 & 30 \\
\hline- Gate & 2.6 & 10 & 20 \\
\hline- Needle & 1 & 10 & 30 \\
\hline- Non-return & 1.5 & 20 & \\
\hline- Plug & 1 & & 20 \\
\hline- Relief & 1 & & 18 \\
\hline- Solenoid & 2 & & 8 \\
\hline
\end{tabular}

\section{Consequence Analysis}

The consequence analysis should cover the sequences that impact performance outcomes in the wake of an initiating or failure event. The objectives of consequence analysis are to assess outcomes of the initiating events that contributed to the risk, and to analyze potential event sequences after the initiating event and assess the magnitude of adverse effects on the environment, humans, and critical infrastructure. The consequence calculations predict the physical circumstances relating specifically to the risk event. The resulting assessments should predict the effects of the scenarios on the subjects for which risk is to be estimated. For the purposes of the current evolution of the technical risk model, the focus of consequence is the unavailability of the unit component to the overall system operation. A metric for this condition is mean time to repair (MTTR), which relies on a maintainability prediction (see Smith 2011 for a detailed description about the well-established methods for predicting maintainability). ${ }^{1}$ Two approaches are commonly employed. First, the work-study method breaks a sampled task into concrete work elements and analyzes each task. The approach is only feasible if extensive data about a large number of tasks on the types of equipment under investigation are gathered and available. The second approach entails the identification of maintainability factors and ranking each factor. The scores are then converted to an MTTR through a nomograph obtained by regression analysis.

\footnotetext{
${ }^{1}$ Smith, DJ. Reliability, Maintainability and Risk: Practical Methods for Engineers. Oxford, UK: Butterworth-
} Heinemann, 2011. 
PNNL-22563

\section{Appendix F: Component Failure Data Estimates}

\section{Piping}

The failure modes for pipe sections can be described as either blockage, leak, or break [Ref. 1 pp. 155156]. In Ref. 1, industry failure data have been used as a basis, supplemented by data from direct experience, and from other literature sources. Data for medium size pipes for general service, in ranges 2 " to 6" and pressures up to $30 \mathrm{~atm}$ are described. A leak is defined here as a small crack or pinhole in the range $0.01 \mathrm{~mm}^{2}$ to $10 \mathrm{~mm}^{2}$. A large leak is in the range $10 \mathrm{~mm}^{2}$ to $1000 \mathrm{~mm}^{2}$. Anything above this range is regarded as a break.

Failure rates for pipes in 2"- 6" class up to 30 atm.

\begin{tabular}{|c|c|}
\hline Failure Mode & Failure Rate \\
\hline Small leak & 0.001 per MHr-meter \\
\hline Large leak & 0.0001 per MHr-meter \\
\hline Break & 0.00003 per MHr-meter \\
\hline
\end{tabular}

An alternative data source is provided by AIChE [Ref. 2, p. 183]. Here catastrophic (i.e., break) pipe failure rates, in units of $\mathrm{MHr}-\mathrm{mile}$, are given as a range:
- Lower
0.000465 per MHr-mile
- Mean
0.0268 per MHr-mile
- Upper
0.104 per MHr-mile

For failures of pipe inlets and outlets with respect to other system components, a range of catastrophic (specifically, leakage greater than $10 \%$ of the flow area) failure rate for metal connections in a piping system is given in Ref. 2 (p.184) as:

- Lower

0.0099 per $\mathrm{MHr}$

- Mean

0.57 per $\mathrm{MHr}$

- Upper

2.20 per $\mathrm{MHr}$

The most important failure modes for flanges connecting piping to other components are leak or breakdown, with many causes [Ref. 1, pp. 156-158].

\section{Failure rates for flanges in 2"-6" piping.}

\begin{tabular}{|l|l|}
\hline Failure Mode & Failure Rate \\
\hline Small leak & 0.4 per $\mathrm{MHr}$ \\
\hline Large leak & 0.03 per $\mathrm{MHr}$ \\
\hline Break & 0.01 per $\mathrm{MHr}$ \\
\hline
\end{tabular}

Welds represent one of the weakest points in piping, and it is probable that many of the failures in piping in fact occur at welds [Ref. 1, pp. 157-158]. The weld failure data below concern high pressure, moderately high temperature steam and water service. This data may be particularly applicable to welds about steam line inlet/outlet situations. 
PNNL-22563

Weld failure rates for larger pipe sizes (1.5" to 24") for high pressure, moderately high temperature steam and water service.

\begin{tabular}{|l|l|}
\hline Failure Mode & Failure Rate \\
\hline Leakage (less than 10\% of weld area) & 0.03 per $\mathrm{MHr}$ \\
\hline Breakage & 0.003 per $\mathrm{MHr}$ \\
\hline
\end{tabular}

The MDT for piping failures can be complicated by many factors, not the least of which is the size and length of pipes and the complexity and accessibility of the piping network. Instead, a model or heuristic for MDT is plausible for estimating MDT, at least in relative terms. A good example is provided by Ref. 3 , where pipe availability indicator models for water distribution networks are presented (equations 3-8). These availability estimates can then be used to find predicted MDTs by dividing the unavailability (1availability) by an appropriate piping failure rate. The data -based analysis [Ref. 3] identifies the Poisson model formulation of availability as providing a lower bound to mechanical reliability (i.e., the upper bound of unavailability).

\section{Valves}

Data exist on a valve-type basis, but there is not sufficient specificity of the components design at this stage of analysis to select one kind over another. The ANSI/IEEE Standard 500-1984 [Ref. 4, p.134] provides a composite failure rate and outage time for a large class of valves.

The failure rate for all modes is in the range:

- Low

- Recommended

- High
0.03 per $\mathrm{MHr}$

1.39 per $\mathrm{MHr}$

3, 230 per $\mathrm{MHr}$

"Out of Service" time is defined as the average time required to get the failure, analyze it, obtain spare parts, repair and return the item or equipment to service, including planned delays. The quoted outage time range is:

- Low

- Recommended

- High
$0.4 \mathrm{Hr}$

$117 \mathrm{Hr}$

$8,140 \mathrm{Hr}$

Additional sources of data are provided in References 2 and 5. Reference 5 has data over types of valves, leading to a composite average range of:
- Low
2 per $\mathrm{MHr}$
- Mean
15 per $\mathrm{MHr}$
- High
20 per $\mathrm{MHr}$

For additional information on the spread of failure rates, Reference 2 (pp. 200-202) has the following data.

For motor-operated valves, the range of catastrophic failure rates (due to "spurious operation") is:

- Lower

0.239 per $\mathrm{MHr}$

- Mean

1.36 per $\mathrm{MHr}$

- Upper

3.80 per $\mathrm{MHr}$

For pneumatic-operated valves, the range of catastrophic failure rates (due to "spurious operation") is: 
PNNL-22563
- Lower
0.274 per $\mathrm{MHr}$
- Mean
3.59 per $\mathrm{MHr}$
- Upper
12.3 per $\mathrm{MHr}$

For solenoid-operated valves, the range of catastrophic failure rates (due to "spurious operation") is:

- Lower

0.108 per $\mathrm{MHr}$

- Mean

0.409 per $\mathrm{MHr}$

- Upper

0.985 per $\mathrm{MHr}$

In addition, for solenoid-operated valves the range of catastrophic failure rates over all modes of failure (external leakage, internal leakage $>10 \%$, spurious operation, and no change on demand) is:
- Lower
0.679 per $\mathrm{MHr}$
- Mean
48.7 per $\mathrm{MHr}$
- Upper
189 per $\mathrm{MHr}$

In addition to these base failure rates, contingencies need to be considered. Day-to-day environmental conditions such as high temperature and vibration do have an effect on the failure rates of the valves. The ANSI/IEE Standard [Ref. 4, p. 37] provides environmental factors to be used in conjunction with the failure rates. These factors are to be multiplied by the device failure rates. For instance, the failure rates of valves in the steam lines would be multiplied by a factor of $\sim 1.5$ as compared to the base rates in the cooling water lines. In addition, the valves in the solid sorbent lines may be multiplied by an even higher factor to account for the impacts of moving solids, entailing additional vibration and erosion effects.

\section{Centrifugal pump}

The process system design specifies the use of an electrically driven centrifugal pump made of steel. The ANSI/IEE Standard [Ref. 4, p. 365] provides composite failure rate data for centrifugal pumps. For catastrophic failure, the failure rate range is:
- Low
0 per $\mathrm{MHr}$
- Recommended
7.07 per $\mathrm{MHr}$
- High
584 per $\mathrm{MHr}$

For degraded performance (e.g., fails to run at rated speed or has an external leak), the failure rate range is:
- Low
0 per $\mathrm{MHr}$
- Recommended
14.7 per $\mathrm{MHr}$
- High
834 per $\mathrm{MHr}$

The "out of service" time range is:
- Low
$1.0 \mathrm{Hr}$
- Recommended
$159 \mathrm{Hr}$
- High
7,930 $\mathrm{Hr}$

Additional data comes from Reference 2 (pp. 192-193) for centrifugal pumps in alternating and running operating modes. For the alternating operation mode, the catastrophic failure rates are:
- Lower
43.3 per $\mathrm{MHr}$
- Mean
292.0 per $\mathrm{MHr}$
- Upper
862.0 per $\mathrm{MHr}$

While the degraded rates are: 
PNNL-22563
- Lower
15.8 per $\mathrm{MHr}$
- Mean
920.0 per $\mathrm{MHr}$
- Upper
3560 per $\mathrm{MHr}$

For the running operational mode, the catastrophic failure rates are:

- Lower 0.812 per $\mathrm{MHr}$

- Mean 104 per $\mathrm{MHr}$

- Upper 450 per $\mathrm{MHr}$

While the degraded rates are:

- Lower 0.417 per $\mathrm{MHr}$

- Mean 24 per $\mathrm{MHr}$

- Upper 92.8 per $\mathrm{MHr}$

These data show that the running operational mode increases the pump reliability relative to the alternating mode.

The above numbers are a composite over all sizes of centrifugal pumps. The process system design indicates that the centrifugal pump for the adsorber cooling water has a significantly larger capacity - on the order of 3,000 gpm - than the pump serving the solid heat exchangers for the regenerator heat balance, which apparently only requires a capacity of about 25 gpm. Reference 4 [pp. 387 and 374] provides failure data for these differing pump flow rates.

For a centrifugal pump capable of 2500 - 9999 gpm, the all modes failure rate range is:

- Low 0.75 per $\mathrm{MHr}$

- Recommended $\quad 1.0$ per $\mathrm{MHr}$

- High 1.25 per $\mathrm{MHr}$

For this large size pump, the out of service data is:

- Low $0 \mathrm{Hr}$

- Recommended $632 \mathrm{Hr}$

- High 7,100 Hr

For a generic centrifugal pump under $500 \mathrm{gpm}$, the all modes failure data is:

- Low 14.9 per $\mathrm{MHr}$

- Recommended $23.2 \mathrm{per} \mathrm{MHr}$

- 25.3 per $\mathrm{MHr}$

For this smaller pump size, the out of service time range is:

- Low $0 \mathrm{Hr}$

- Recommended $575 \mathrm{Hr}$

- High 7,930 Hr

Flow and level controls

In the material transport lines for gas, water, steam, and sorbent there will need to be subsystems to monitor and control flow rates or levels through the actuation of valves or other devices. Such systems usually contain, in addition to the controller, a sensor, an indicator, a transmitter or a switch.

Reference 2 (p. 176) provides catastrophic failure rate data for generic controllers:
- Lower
2.43 per $\mathrm{MHr}$ 
PNNL-22563

- Mean

68.8 per $\mathrm{MHr}$

- Upper

260 per $\mathrm{MHr}$

Reference 5 provides controller failure rate ranges for both level and flow instruments, and the composite range for the controller is 5-50 per MHr. For a flow instrument (containing a controller, sensor, transmitter, and switch), the failure rate range is 110-310 per MHr. For a level instrument (containing a controller, indicator, transmitter, and switch), the failure rate range is 15-70 per MHr.

Data on the MDT for flow and level controls has need been found to date. Based on discussion with LLNL maintainability personnel, such controllers should be readily available off-the-shelf, and are usually replaced as plugged-in units (rather that repaired and replaced). The outage time should only be on the order of a day, unless there are unplanned delays that can last up to two weeks. Thus, a plausible MDT range is $8-80 \mathrm{Hr}$.

\section{Adsorber structures}

The passive mechanical structures of the adsorber may be considered as a combination of a metallic, closed, process vessel with tubed and baffled internal heat transfer devices. Even though the adsorber operates at atmospheric pressure, the substantial gas and sorbent mass flow (sorbent flows at about 420 tons per hour), as well as the intrinsic solids holdup (on the order of one to two metric tons), apply pressure to the vessel.

Reference 1 [pp. 158-160] provides some information on pressure vessel failure causes, e.g., overload, material and manufacturing faults, corrosion/erosion, creep or fatigue, excessive vibration, and expansion and contraction. Based on several studies of pressure vessel failure rate data, Reference 1 provides a catastrophic failure rate of 0.00025 per $\mathrm{MHr}$, and a small leak or small break rate of 0.0025 per MHr.

Reference 2 [pp. 203,205] also provide failure data on process vessels. For a generic pressurized metallic vessel the catastrophic failure rate spectrum is:

- Lower 0.000142 per $\mathrm{MHr}$

- Mean 0.0109 per MHr

- Upper 0.0424 per $\mathrm{MHr}$

For a degraded condition of restricted flow, the failure rate range is:
- Lower
0.000951 per $\mathrm{MHr}$
- Mean
0.0636 per MHR
- Upper
0.247 per $\mathrm{MHr}$

For comparison, the catastrophic failure data for a metallic process vessel designed for atmospheric pressures are:
- Lower
0.127 per $\mathrm{MHr}$
- Mean
0.985 per $\mathrm{MHr}$
- Upper
3.02 per $\mathrm{MHr}$

Note that the failure rates are higher for the non-pressurized vessel than the pressurized, since it is not designed and constructed to the same high standards of the P\&V code.

In lieu of more detailed design analysis, the lower and upper heat exchanger apparatuses in the adsorber each can be represented by a generic, indirect contact, heat transfer system of tubes and baffles. Such a surrogate is provided by the equipment of Reference 2, p. 182. This equipment entails an inlet and outlet of cooling water with the heat exchange tubes; and an inlet and outlet of the sorbent with the baffles. The catastrophic (internal leakage or rupture) failure estimates are: 


$\begin{array}{lll}\text { - } & \text { Lower } & 0.0173 \text { per } \mathrm{MHr} \\ \text { - Mean } & 31.1 \text { per } \mathrm{MHr} \\ \text { - Upper } & 131 \text { per } \mathrm{MHr}\end{array}$

The MDT associated with an adsorber vessel or heat exchanger failure is going to be substantial, for a number of reasons. The adsorber structures most likely cannot be repaired in place given their size and configurations. Leaks or ruptures cannot just be patched; rather the vessel or heat exchange components will have to be replaced. Before doing so, the sorbent material has to be removed from the structures. Since the failure is 'unexpected', replacement parts, usually available for planned maintenance, will probably not be readily acquired given their unique size and cost profiles. Replacement of the adsorber unit or its subsystems also impacts many collateral infrastructure and sub-system functions that have to be ameliorated. Thus, a plausible MDT range is $700-8,500 \mathrm{Hr}$.

\section{Cyclone unit}

To date, reliability data on cyclone units has not been forthcoming. As a surrogate, data on motor-driven fan systems may be considered. A good example is provided in Reference 2 [p. 191]. A process or flow goes in and out of a fan module driven by a motor with controls. The failure rate spectrum is:
- Lower
1.75 per $\mathrm{MHr}$
- Mean
9.09 per $\mathrm{MHr}$
- Upper

$$
24.7 \text { per } \mathrm{MHr}
$$

These numbers should be multiplied by an additional factor to account for the substantial erosions that can occur in a particulate cyclone unit and for the unscheduled unit shutdowns [Ref. 6]. The MDT for a cyclone unit can be substantial and depends on whether parts of the cyclone are being replaced or repaired or the whole unit is being replaced. The range of MDT is plausibly $40-700 \mathrm{Hr}$.

\section{Carbon loading}

The estimation of the failure rate for carbon loading - adsorption - is based on an uncertainty quantification (UQ) study of CO2 capture in the adsorber ( $\mathrm{Ng}$, et al., 2012) ${ }^{1}$ combined with input from the TRL Uncertainty Model. The UQ study considered the frequency distribution of CO2 capture in the adsorber based on sample variations in the values of chemical reaction parameters calibrated to TGA data (ibid., pp. 4-25 to 4-28). This distribution had a mean of $88.5 \%$ capture with a standard deviation of 3.12 ; and hence a variability [(std. dev)/mean] of $3.5 \%$. The uncertainty bounds were $76 \%$ to $94 \%$. Furthermore, the distribution gives a 0.45 proportion of cases of carbon loading being greater than or equal to the target $90 \%$ standard.

This threshold fraction can be modeled (Coles, 2004) ${ }^{2}$ based on Poisson assumptions (randomness, constant failure rate) for adsorption success or 'reliability' -- defined as being greater than or equal to $90 \%$ capture -- as:

$$
R=e^{-\lambda t}
$$

where $\lambda$ is a constant failure rate. For $R=0.45, \lambda t=0.8$. To estimate the failure rate, the time over which this fraction is likely needs to be approximated (The UQ study was based on steady state conditions and did not consider this question).

\footnotetext{
${ }^{1} \mathrm{Ng}$, B., C. Tong, J. Leek, J.Ou (October 2012). "Uncertainty Quantification of the A650.1 Process Model”, Lawrence Livermore National Laboratory.

${ }^{2}$ Coles, S. (2004). An Introduction to Statistical Modeling of Extreme Values, Chapter 4. Springer Series in Statistics.
} 
The process optimization effort (Kim, 2012) ${ }^{1}$ used a sorbent loss fraction of $0.005 \%$ per adsorber cycle and 40 such cycles a day; or an attrition of $0.2 \%$ per day. A variation or loss of $3.5 \%$ should assure a failure fraction of 0.55 based on the distribution in the UQ study. This value corresponds to 17.5 days. However, for such an immature sorbent technology the uncertainty from the adsorber's TRL needs to be accounted. For a TRL in the 3-5 range, the TRL Uncertainty Model shows that an uncertainty multiplier of 1.75 bounds the bulk of the likely uncertainty (see Appendix G). Thus, the time may be approximated to be on the order of 10 days. This result corresponds to a failure rate of about 3,300 failures per $\mathrm{MHr}$.

\section{References}

[1] Taylor, J.R. (1994); Risk Analysis for Process Plant, Pipelines and Transport, Taylor and Francis, London. [2] American Institute of Chemical Engineers (1989); Guidelines for Process Equipment Reliability Data with Data Tables (published online September 28, 2010; Online ISBN: 9780470938355).

[3] Tabesh, M., J. Soltani, R. Farmani, and D. Savic (2009); "Assessing pipe failure rate and mechanical reliability of water distribution networks using data driven modeling", Journal of Hydroinformatics, 11.1 (doi:

10.2166/hydro.2009.008).

[4] ANSI/IEEE (1986); Std. 500-1984 P\&V, Reliability Data for Pumps and Drivers, Valve Actuators, and Valves. [5] Smith, D.J. (2005); Reliability, Maintainability and Risk: Practical Methods for Engineers, Seventh Edition. Elsevier. Also, FARADIP.THREE database.

[6] Chen Y-M., M. Nieskins, S.B. Reddy Karri, and T.M. Knowlton, "Developments in cyclone technology improve FCC unit reliability", http://www.digitalrefining.com/data/articles/file/802143366.pdf

\footnotetext{
${ }^{1}$ Kim, H. (February 2012). "Result of Simulation-based Optimization of Hybrid System”, National Energy Technology Laboratory.
} 
PNNL-22563

\section{Appendix G: TRL Uncertainty Modeling}

Typically, uncertainty quantification and risk analysis focuses on aleatory uncertainties (probabilistic uncertainties/risks). These are uncertainties due to the randomness of the model inputs (environment). Another type of uncertainty is the uncertainty due to gaps in knowledge or lack of technology maturity, also called epistemic uncertainty. Our risk analysis team has focused on investigating the relation of epistemic uncertainties to measures such as the technical readiness level (TRL). This effort is reflected in the TRL modeling approach to developing stochastic measures associated with positions in the TRL spectrum. The hypothesis is that the uncertainty reduces with TRL according to a distribution (e.g., log normal) and thus results in a distribution of uncertainty bias (multipliers) for a given TRL state. The following information is a condensed version of Engel at al. (2012). ${ }^{1}$

We compute uncertainty as cumulative probability distributions across all relevant TRLs with uncertainty bounds to estimate and visualize the uncertainty by transforming qualitative assessment provided by subject matter experts through an elicitation questionnaire into a quantitative assessment. We use the count of $\mathrm{Y} / \mathrm{N}$ questionnaire responses for each of the 9 readiness levels and the binomial distribution to construct a likelihood model as follows:

$$
P\left(T R L_{i}\right)=\left(\begin{array}{l}
n \\
k
\end{array}\right) p^{k}(1-p)^{n-k}
$$

Where $n$ is the number of questions for each specific TRL $(i), k$ is the number of "Yes" for each TRL, and $p$ is the probability parameter (set to 0.5 , which assumes that it is equally likely to get a yes or no for each question). Equation G.1 calculates the probability or likelihood of a technology of being through a TRL, based on the number of questions that got a Yes answer. Notional results for the current hybrid solid sorbent system from the TRL Likelihood Model (TRL-LM) are show in Figure G.1.

The next step in our process is to estimate uncertainty due to the lack of technology maturity. It is desirable for carbon capture technologies to progress to a higher TRL, since the uncertainty bound should decrease as the TRL increases, as shown in Figure G.2. A conservative approach for this modeling would be to use the uncertainty bound at the largest TRL that satisfies $(P(T R L)=1.0)$. However, this approach ignores any information that may be gained from the tasks already completed at higher TRLs. Therefore, we model the uncertainties based on the TRL as a mixture problem based on an estimate for the probability of completing each level $\left(\alpha_{i}\right)$ and the uncertainty (CDF) for each level, $\mathrm{F}_{i}(x)$. Assuming a linear mixture problem of each level, the uncertainty $(\mathrm{U})$ is estimated as follows:

$$
U=\gamma_{1} F\left(x_{1}\right)+\gamma_{2} F\left(x_{2}\right)+\ldots+\gamma_{9} F\left(x_{9}\right)
$$

Typically, in a mixture problem the weights $\left(\gamma_{i}\right)$ sum to 1.0. However, in our example (Figure G.2) the weights for the adsorber sum to 3.7. Also, since we have already satisfied the criteria for completing TRL 1-3, we can ignore the uncertainty estimates for TRL 1 and 2. Therefore, Equation G.2 can be modified as shown in Equation G.3.

$$
U=\left(1-\alpha_{4}\right)\left(1-\alpha_{5}\right) F\left(x_{3}\right)+\alpha_{4}\left(1-\alpha_{5}\right) F\left(x_{4}\right)+\alpha_{5} F\left(x_{5}\right)
$$

Applying Equation G.3 to the results from the TRL-LM (Figure G.2), we get the CDF shown in Table G.1.

\footnotetext{
${ }^{1}$ Engel, etl al. (2012). Development of Technology Readiness Level (TRL) Metrics and Risk Measures, PNNL21737, available at www.osti.gov/bridge/product.biblio.jsp?osti_id=1067968.
} 
PNNL-22563

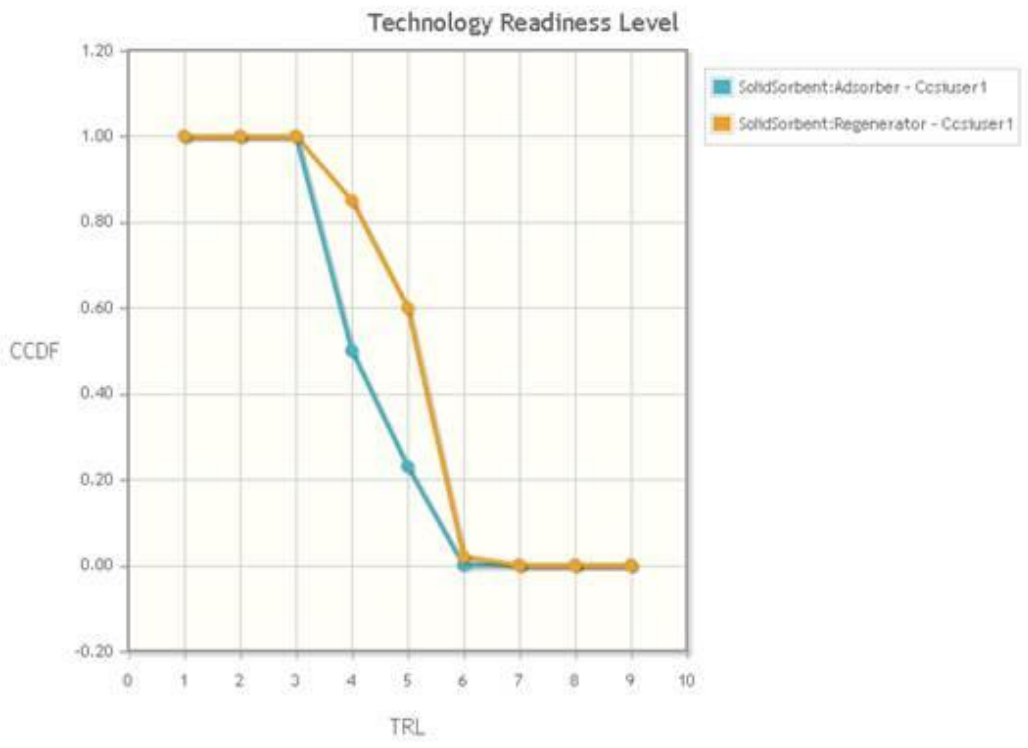

\begin{tabular}{|c|c|c|c|c|c|c|c|c|c|c|c|}
\hline \multirow[b]{2}{*}{ Technologyes } & \multirow[b]{2}{*}{ Process } & \multirow[b]{2}{*}{ Statistics } & \multirow[b]{2}{*}{ TRL1] } & \multirow[b]{2}{*}{ TRL2: } & \multirow[b]{2}{*}{ TRL3X] } & \multirow[b]{2}{*}{ TRLAS } & \multirow[b]{2}{*}{ TRLER } & \multirow[b]{2}{*}{ TRL6E } & \multicolumn{3}{|c|}{ Download results as .CSV fill } \\
\hline & & & & & & & & & TRL7DS & TRL8ES & TRLOA \\
\hline SolidSorbent & Adsorber & Ccsiuser1 & 1.00 & 1.00 & 1.00 & 0.50 & 0.23 & 0.00 & 0.00 & 0.00 & 0.00 \\
\hline SolidSorbent & Regenerator & Cosiuser1 & 1.00 & 1.00 & 1.00 & 0.85 & 0.60 & 0.02 & 0.00 & 0.00 & 0.00 \\
\hline
\end{tabular}

Figure G.1 Notional TRL-LM results for the current hybrid solid sorbent system
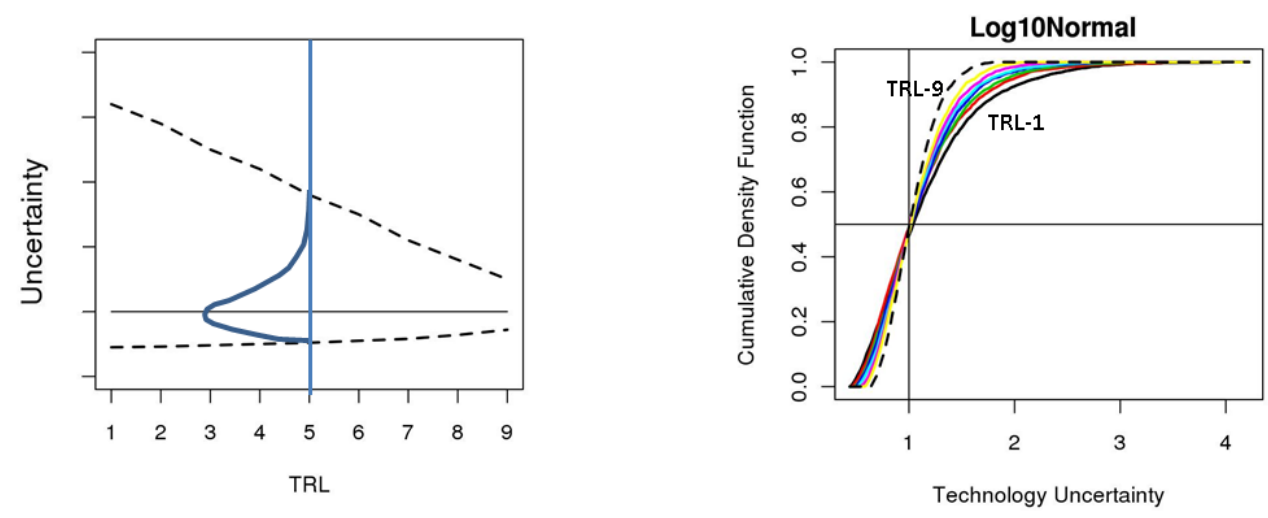

Figure G.2 TRL uncertainty estimation (left side) and CDF (right side) assuming uncertainties are distributed as a Log Normal

Table G.3 Cumulative distribution of uncertainties for the TR Uncertainty Model

$\begin{array}{ccccccccc}\text { TRL1 } & \text { TRL2 } & \text { TRL3 } & \text { TRL4 } & \text { TRL5 } & \text { TRL6 } & \text { TRL7 } & \text { TRL8 } & \text { TRL9 } \\ 0.000 & 0.000 & 0.385 & 0.770 & 1.000 & 1.000 & 1.000 & 1.000 & 1.000\end{array}$


PNNL-22563

Now, utilizing results (CDF) from Table G.1 and Figure G.2, we can simulate results from the TRL Uncertainty Model (TRL-UM). These results are shown in Figure G.3 and are used in the technical and financial risk models.

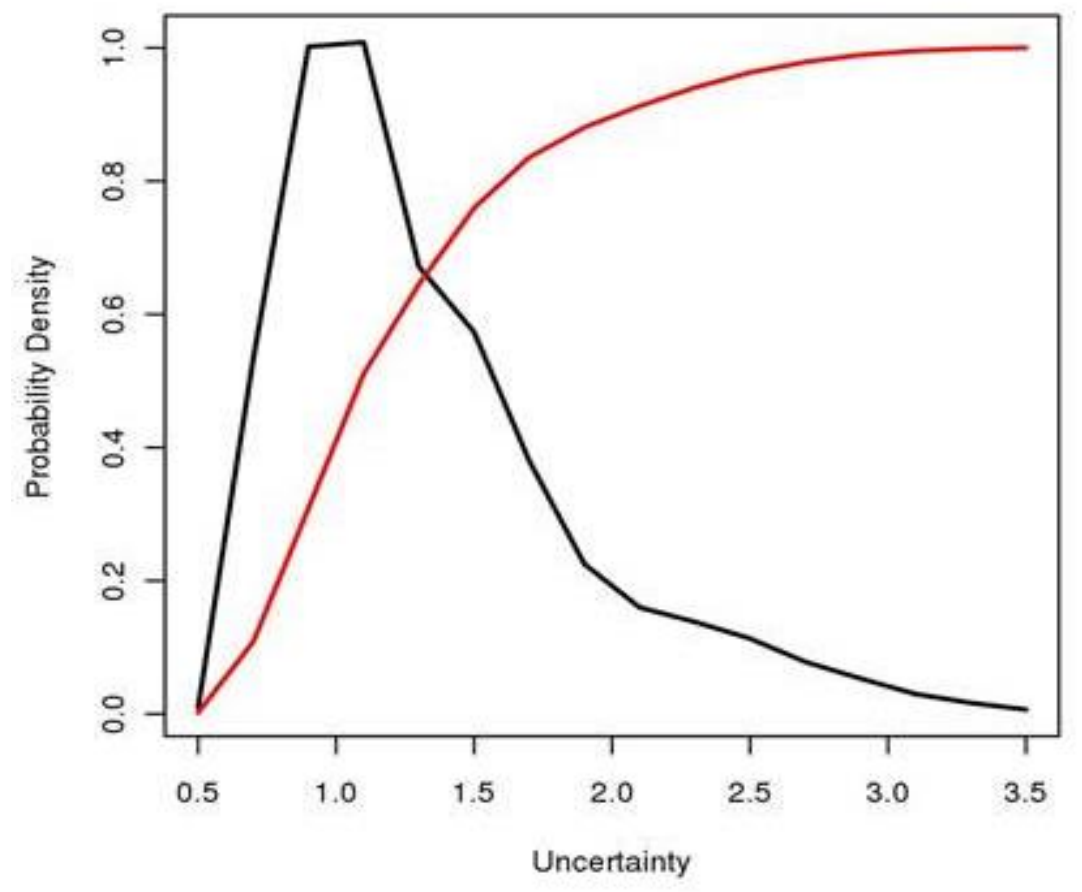

Figure G.3 Uncertainty estimations (probability density) utilizing results from the TRL-LM and TRL-UM 



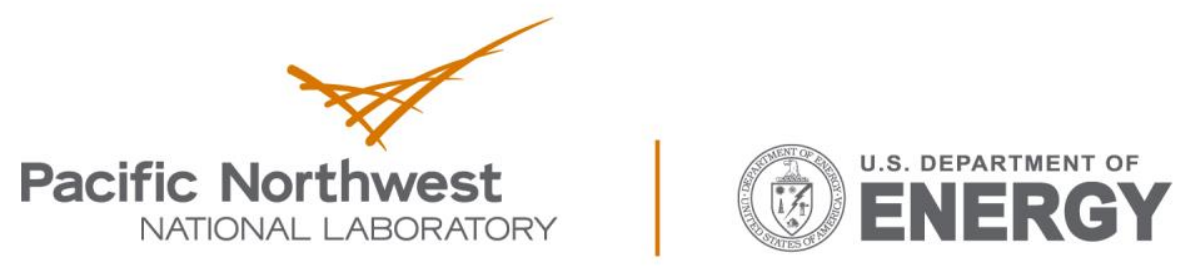

Proudly Operated by Battelle Since 1965

902 Battelle Boulevard

P.O. Box 999

Richland, WA 99352

1-888-375-PNNL (7665)

www.pnnl.gov 